\title{
Advanced Background Subtraction Applied to Aeroacoustic Wind Tunnel Testing
}

\author{
Christopher J. Bahr* \\ NASA Langley Research Center, Hampton, Virginia 23681 \\ William C. Horne ${ }^{\dagger}$ \\ NASA Ames Research Center, Moffett Field, California 94035
}

\begin{abstract}
An advanced form of background subtraction is presented and applied to aeroacoustic wind tunnel data. A variant of this method has seen use in other fields such as climatology and medical imaging. The technique, based on an eigenvalue decomposition of the background noise cross-spectral matrix, is robust against situations where isolated background autospectral levels are measured to be higher than levels of combined source and background signals. It also provides an alternate estimate of the cross-spectrum, which previously might have poor definition for low signal-to-noise ratio measurements. Simulated results indicate similar performance to conventional background subtraction when the subtracted spectra are weaker than the true contaminating background levels. Superior performance is observed when the subtracted spectra are stronger than the true contaminating background levels. Experimental results show limited success in recovering signal behavior for data where conventional background subtraction fails. They also demonstrate the new subtraction technique's ability to maintain a proper coherence relationship in the modified cross-spectral matrix. Beamforming and deconvolution results indicate the method can successfully separate sources. Results also show a reduced need for the use of diagonal removal in phased array processing, at least for the limited data sets considered.
\end{abstract}

\section{Nomenclature}

$\begin{array}{ll}\mathbf{B} & =\text { prewhitening operator } \\ b & =\text { background pressure signal } \\ \text { CSM } & =\text { cross-spectral matrix, general acronym } \\ d & =\text { background pressure and electronic signals } \\ f & =\text { frequency } \\ \mathbf{G} & =N \times N \text { cross-spectral matrix } \\ \widehat{\mathbf{G}} & =\text { prewhitened } N \times N \text { cross-spectral matrix } \\ G & =\text { autospectrum } \\ H & =\text { Hermitian }(\text { conjugate) transpose } \\ \mathbf{I} & =\text { identity matrix } \\ i, j & =\text { indices } \\ M & =\text { Mach number } \\ N & =\text { microphone count } \\ n & =\text { electronic noise } \\ p & =\text { pressure } \\ s & =\text { signal of interest } \\ t & =\text { time } \\ \mathbf{X} & =\text { matrix of eigenvectors of } \boldsymbol{G}\end{array}$

*Aerospace Engineer, Aeroacoustics Branch, MS 461, Senior Member AIAA, christopher.j.bahr@nasa.gov

$\dagger$ Aerospace Engineer, Experimental Aero-Physics Branch, Associate Fellow AIAA 


$$
\begin{array}{ll}
\widehat{\mathbf{X}} & =\text { matrix of eigenvectors of } \widehat{\boldsymbol{G}} \\
\mathbf{x} & =\text { eigenvector of } \boldsymbol{G} \\
\gamma^{2} & =\text { coherence-squared } \\
\boldsymbol{\Lambda} & =\text { matrix of eigenvalues of } \boldsymbol{G} \\
\widehat{\boldsymbol{\Lambda}} & =\text { matrix of eigenvalues of } \widehat{\boldsymbol{G}} \\
\lambda & =\text { eigenvalue }
\end{array}
$$

\section{Introduction}

B ACKGROUND noise contamination can be a significant problem in aeroacoustic wind tunnel testing. In In open test section wind tunnels, transient wind gusts may impinge on microphones and contaminate measurements. ${ }^{[2}$ In both tunnel types, facility background acoustic noise will be present in the measurements. If the acoustic signals of interest are stationary and random, techniques exist which can separate contamination by spurious transient signals. ${ }^{2}$ However, stationary contamination from steady turbulent flow over microphones and facility acoustic sources remains.

If the microphone data are used for beamforming or deconvolution analysis, diagonal removal is a means of overcoming microphone "self-noise." 11 With diagonal removal, the covariance or cross-spectral matrix (CSM) of the microphone signal's narrowband Fourier coefficients is computed. The diagonal of this matrix, containing the autospectral terms, is set to zero and the beamforming algorithm adjusted appropriately. This technique removes, from the beamforming analysis, any contamination which is uncorrelated between microphones. However, it has no mechanism for analyzing individual microphone autospectra, and does not remove correlated contamination such as that due to facility acoustic sources.

Background subtraction is a technique which can remove correlated contamination. With background subtraction, a reference or tare measurement is taken without the aeroacoustic source of interest present. The autospectrum or CSM of this tare measurement is subtracted from the autospectrum or CSM of the installed aeroacoustic source ${ }^{[3}$ If the facility acoustic sources do not change between the tare and installed measurements and the facility acoustic sources are uncorrelated with the aeroacoustic source of interest, this subtraction can significantly improve the estimate of an aeroacoustic source ${ }^{4}$ However, if the facility background noise decreases in level during the installed measurement non-physical negative powers may be computed.2] Additionally under such situations the meaning of cross-spectral levels becomes ambiguous ${ }^{2 / 5}$

Many methods have been proposed to provide more robust handling of background noise contamination. Adaptive filtering is one option which may be used if a reference measurement is available ${ }^{[6}$ Least-squares modeling using a tare CSM is another ${ }^{7}$ Eigenvalue analysis, where the eigenvalues and eigenvectors of the CSM related to the desired aeroacoustic sources are separated from those related to the background contamination, has been considered in many situations. $[8]$ Identification of the appropriate signal and background eigenvalues and eigenvectors may require some a-priori knowledge or data assumptions, although more advanced analysis methods have been proposed 12

The proposed method in this work follows such a path, with the objective of implementing a straightforward eigenvector identification and removal technique. It is similar to prewhitening noise identification and removal methods used with covariance matrices in fields such as climatology ${ }^{13}$ and biomedical imaging! 14 Derivation and implementation of both conventional background subtraction and the new method are first provided. Analysis with simulated acoustic data superimposed on background data from a real wind tunnel test is then presented. Finally, experimental data from both closed-wall and open-jet wind tunnel tests are processed with the technique.

\section{Traditional Background Subtraction}

For a traditional acoustic measurement of stochastic stationary signals, a pressure measurement at a microphone can be decomposed into three components,

$$
p_{i}(t)=p_{i, s}(t)+p_{i, b}(t)+n_{i}(t) .
$$

Here, $p_{i, s}(t)$ is the acoustic signal of interest at the microphone, $p_{i, b}(t)$ comprises additional acoustic and hydrodynamic background pressure fluctuations observed by the microphone, and $n_{i}(t)$ is electronic noise 
contaminating the microphone measurement. Assuming that $p_{i, s}(t), p_{i, b}(t)$, and $n_{i}(t)$ are all mutually uncorrelated and stationary, the steady-state autospectrum of microphone $i$ is expressed as

$$
G_{i i}(f)=G_{i i, s}(f)+G_{i i, b}(f)+G_{i i, n}(f) .
$$

This can be generalized to the cross-spectrum between microphones $j$ and $i$ as

$$
G_{j i}(f)=G_{j i, s}(f)+G_{j i, b}(f)+G_{j i, n}(f) .
$$

Often, $G_{j i, n}(f)$ is assumed to be zero for $j \neq i$. However, this may not always be the case if coherent electromagnetic interference is present in the measurement. This assumption is not a requirement for background subtraction and is not made here. For $N$ microphones, the CSM is thus

$$
\mathbf{G}(f)=\mathbf{G}_{s}(f)+\mathbf{G}_{b}(f)+\mathbf{G}_{n}(f) .
$$

Subsequent frequency dependence is suppressed in the notation.

$\mathbf{G}_{s}$ is the quantity of interest in aeroacoustic analysis. A background measurement can be conducted to acquire a background CSM,

$$
\mathbf{G}_{d}=\mathbf{G}_{d, b}+\mathbf{G}_{d, n} .
$$

Assuming the background and electronic statistics are completely unchanged from the background measurement to the signal measurement, then $\mathbf{G}_{d, b}=\mathbf{G}_{b}$ and $\mathbf{G}_{d, n}=\mathbf{G}_{n}$, so

$$
\mathbf{G}=\mathbf{G}_{s}+\mathbf{G}_{d}
$$

and

$$
\mathbf{G}_{s}=\mathbf{G}-\mathbf{G}_{d} .
$$

Eq. (7) is the traditional background subtraction equation. When all of the assumptions hold true, this will recover the CSM of the signal of interest. However, any changes in background statistics will lead to errors. Direct subtraction may overpredict acoustic levels or predict negative autospectral levels, depending on how the background signals change between measurements. Depending on the signal-to-noise ratio and various source relationships, any number of errors may manifest in the magnitude and phase of cross-spectral terms when the background measurement does not accurately represent background levels during the aeroacoustic source measurement. These changes are particularly problematic because no obvious means of identifying errors are present, as opposed to the obvious error with negative autospectral levels. Finally, direct matrix subtraction may yield an estimate of $\mathbf{G}_{s}$ which is not positive semidefinite, which may have negative implications in subsequent data analysis.

\section{Eigenvalue Identification and Subtraction}

The following derivation is adapted from work in climatology ${ }^{13}$ and biomedical imaging 14 where the method is applied to time-delay covariance matrices. A CSM is a covariance matrix of narrowband Fourier coefficients for a given frequency. This makes it a Hermitian, positive semidefinite matrix. The eigenvalues of a Hermitian matrix, $\lambda_{i}$, are real. The eigenvalues of a Hermitian, positive semidefinite matrix are greater than or equal to zero, $\lambda_{i} \geq 0$. The matrix of eigenvectors of a Hermitian matrix, $\mathbf{X}$, is unitary $\frac{15}{6}$ The eigenvalue decomposition of the CSM is thus

$$
\mathbf{G}_{d}=\mathbf{X}_{d} \boldsymbol{\Lambda}_{d} \mathbf{X}_{d}^{H},
$$

with $\boldsymbol{\Lambda}$ a size $N \times N$ real diagonal matrix containing the eigenvalues $\lambda_{i, d} . \mathbf{X}_{d}$, size $N \times N$, has the property $\mathbf{X}_{d}^{H} \mathbf{X}_{d}=\mathbf{I}=\mathbf{X}_{d} \mathbf{X}_{d}^{H}$.

Using this definition, an inverse matrix $\boldsymbol{\Lambda}_{d}^{-1 / 2}$ can be constructed. This matrix is the square root of the inverse of $\boldsymbol{\Lambda}_{d}$. It is a real, diagonal matrix, with elements $1 / \sqrt{\lambda_{i}}$ for $\lambda_{i}>0$ and zero for $\lambda_{i}=0$. Truncating $\lambda_{i}=0$ terms, $\boldsymbol{\Lambda}_{d}^{-1 / 2} \boldsymbol{\Lambda}_{d} \boldsymbol{\Lambda}_{d}^{-1 / 2}=\mathbf{I}$. Eq. (8) can then be manipulated (truncating $\mathbf{X}$ to remove columns associated with $\lambda_{i}=0$ terms) to yield

$$
\boldsymbol{\Lambda}_{d}^{-1 / 2} \mathbf{X}_{d}^{H} \mathbf{G}_{d} \mathbf{X}_{d} \boldsymbol{\Lambda}_{d}^{-1 / 2}=\mathbf{I}
$$


Defining $\mathbf{B}_{d}=\mathbf{X}_{d} \boldsymbol{\Lambda}_{d}^{-1 / 2}$ as a "prewhitening" operator simplifies Eq. (9) to

$$
\mathbf{B}_{d}^{H} \mathbf{G}_{d} \mathbf{B}_{d}=\mathbf{I} .
$$

The operator can be applied to Eq. (6) and a prewhitened CSM defined as $\widehat{\mathbf{G}}=\mathbf{B}_{d}^{H} \mathbf{G B}_{d}$, yielding

$$
\widehat{\mathbf{G}}=\widehat{\mathbf{G}}_{s}+\mathbf{I} \text {. }
$$

The relationship in Eq. (11) could be leveraged in direct subtraction to recover the signal CSM. However, this may suffer from similar problems as standard CSM background subtraction. It is desirable to find a means to construct an estimate of $\mathbf{G}_{s}$ which maintains this property. One way to do so is through further eigenvalue analysis.

In general, eigenvalue analysis cannot be used to directly separate the matrices in Eq. 66. However, it can be used to separate the matrices in the prewhitened case of Eq. (11). Specifically, properties of the identity matrix and its effect on the eigenvalues and eigenvectors of another matrix in matrix addition can be leveraged. An eigendecomposition of $\widehat{\mathbf{G}}$ can be performed as

$$
\widehat{\mathbf{G}}=\widehat{\mathbf{X}} \widehat{\mathbf{\Lambda}} \widehat{\mathbf{X}}^{H} \text {. }
$$

As stated previously, $\mathbf{G}$ is Hermitian and positive semidefinite. Since $\mathbf{B}_{d}$ is invertible, this means $\widehat{\mathbf{G}}$ is also positive semidefinite. The same holds for $\widehat{\mathbf{G}}_{s}$. An eigendecomposition of Eq. (11) then yields

$$
\widehat{\mathbf{X}} \widehat{\mathbf{\Lambda}} \widehat{\mathbf{X}}^{H}=\widehat{\mathbf{X}}_{s} \widehat{\boldsymbol{\Lambda}}_{s} \widehat{\mathbf{X}}_{s}^{H}+\mathbf{I}=\widehat{\mathbf{X}}_{s} \widehat{\boldsymbol{\Lambda}}_{s} \widehat{\mathbf{X}}_{s}^{H}+\widehat{\mathbf{X}}_{s} \mathbf{I} \widehat{\mathbf{X}}_{s}^{H}=\widehat{\mathbf{X}}_{s}\left(\widehat{\boldsymbol{\Lambda}}_{s}+\mathbf{I}\right) \widehat{\mathbf{X}}_{s}^{H}
$$

which holds due to the nature of the identity matrix and the distributive property of matrix multiplication. What this suggests is that the eigenvectors of $\widehat{\mathbf{G}}$ are the eigenvectors of $\widehat{\mathbf{G}}_{s}$, such that $\widehat{\mathbf{X}}=\widehat{\mathbf{X}}_{s}$. Additionally it shows that the eigenvalues of $\widehat{\mathbf{G}}$ are related to the eigenvalues of $\widehat{\mathbf{G}}_{s}$ by $\widehat{\lambda}_{i}=\widehat{\lambda}_{i, s}+1$. Since $\widehat{\mathbf{G}}_{s}$ is Hermitian and positive semidefinite, this means that the eigenvalues of $\widehat{\mathbf{G}}$ must be greater than or equal to unity. Eigenvalues greater than unity correspond to eigenvalue-eigenvector pairs of the signal $\widehat{\mathbf{G}}_{s}$, while those equal to unity correspond to eigenvalue-eigenvector pairs of the noise. Therefore, an estimate of $\widehat{\mathbf{G}}_{s}$ can be made by performing an eigenvalue decomposition of $\widehat{\mathbf{G}}$, subtracting the identity matrix from $\widehat{\boldsymbol{\Lambda}}$, retaining only the subspace of resultant eigenvalues greater than zero, and reconstructing a CSM based on that subspace. The signal estimate can then be transformed back to the real signal domain by

$$
\mathbf{G}_{s}=\left(\mathbf{B}_{d}^{-1}\right)^{H} \widehat{\mathbf{G}}_{s} \mathbf{B}_{d}^{-1},
$$

where $\mathbf{B}_{d}^{-1}=\boldsymbol{\Lambda}_{d}^{1 / 2} \mathbf{X}_{d}^{H}$. This estimate of $\mathbf{G}_{s}$ is Hermitian and positive semidefinite. To re-iterate, the proposed method uses the following procedure:

- Measure both $\mathbf{G}$ and $\mathbf{G}_{d}$.

- Compute $\mathbf{B}_{d}$ based on the eigendecomposition of $\mathbf{G}_{d}$. Note that experimental and numerical realities may lead to some of the eigenvalues of $\mathbf{G}_{d}$ being excessively small, zero, or slightly less than zero. This necessitates a choice in an acceptable threshold in the construction of $\mathbf{B}_{d}$. Eigenvalues below this threshold, either due to their magnitude or possibly being computed as slightly negative, are discarded along with their corresponding eigenvectors. An alternative is to slightly perturb $\mathbf{G}_{d}$ to enforce positive semi-definite behavior and retain all positive eigenvalues. Methods exist for finding the nearest positive semi-definite matrix in terms of the Frobenius norm $\frac{16}{16}$ A simple MATLAB implementation of the referenced method is used in this work ${ }^{17}$

- Compute $\widehat{\mathbf{G}}$ using $\mathbf{G}$ and $\mathbf{B}_{d}$.

- Compute $\widehat{\boldsymbol{\Lambda}}$ and $\widehat{\mathbf{X}}$ from the eigendecomposition of $\widehat{\mathbf{G}}$.

- Compute $\widehat{\boldsymbol{\Lambda}}_{s}=\widehat{\boldsymbol{\Lambda}}-\mathbf{I}$, retaining only the positive eigenvalues. This leads to a dimension reduction of $\widehat{\Lambda}_{s}$ from $\widehat{\Lambda}$.

- Define $\widehat{\mathbf{X}}_{s}=\widehat{\mathbf{X}}$, while retaining only the eigenvector columns associated with the retained eigenvalues of $\widehat{\Lambda}_{s}$.

- Compute $\widehat{\mathbf{G}}_{s}=\widehat{\mathbf{X}}_{s} \widehat{\boldsymbol{\Lambda}}_{s} \widehat{\mathbf{X}}_{s}^{H}$.

- Use $\mathbf{B}_{d}^{-1}$ to invert the prewhitening transformation and recover a rank-reduced estimate of $\mathbf{G}_{s}$. 


\section{Perturbations of Background Sources}

For ideal measurements where $\mathbf{G}_{d}$ does not change from background measurement to signal measurement, both methods of background subtraction work. The potential failings of traditional background subtraction when $\mathbf{G}_{d}$ does change have been discussed. These are now considered for the alternate method. Sekihara et al ${ }^{14}$ address this by first assuming the signal and background subspaces are orthogonal, and then discussing an error term for when they are not. This work will only address the former situation, thus neglecting interactions between background and aeroacoustic sources.

Four perturbation scenarios can be considered. In the first, a source which is not present in the separate background measurement appears in the background of the aeroacoustic source measurement. Assuming that this source exists in a subspace orthogonal to the background measurement subspace, the result is trivial. This background source will be treated as an aeroacoustic source and remain, contaminating the estimate of $\mathbf{G}_{s}$. The second and third scenarios involve background sources either growing or weakening in strength between the background and aeroacoustic measurements. Both can be addressed in a single discussion. The fourth, where a background source disappears prior to the aeroacoustic measurement, will be considered as a subset of the weakening of a background source when the source strength goes to zero.

For the four scenarios considered, a background measurement is conducted and $\mathbf{B}_{d}$ computed. Background source $i$ has a magnitude change of $\Delta \lambda_{i}$ between the background and aeroacoustic measurements. The aeroacoustic measurement CSM is thus expressed as

$$
\mathbf{G}=\mathbf{G}_{s}+\mathbf{G}_{d}+\Delta \lambda_{i} \mathbf{x}_{i} \mathbf{x}_{i}^{H} .
$$

Multiplying by the prewhitening operator and its conjugate transpose yields

$$
\widehat{\mathbf{G}}=\widehat{\mathbf{G}}_{s}+\mathbf{I}+\Delta \lambda_{i} \mathbf{B}_{d}^{H} \mathbf{x}_{i} \mathbf{x}_{i}^{H} \mathbf{B}_{d} .
$$

The last term of Eq. (16) can be expanded as

$$
\Delta \lambda_{i} \mathbf{B}_{d}^{H} \mathbf{x}_{i} \mathbf{x}_{i}^{H} \mathbf{B}_{d}=\Delta \lambda_{i} \boldsymbol{\Lambda}_{d}^{-1 / 2} \mathbf{X}_{d}^{H} \mathbf{x}_{i} \mathbf{x}_{i}^{H} \mathbf{X}_{d} \boldsymbol{\Lambda}_{d}^{-1 / 2} .
$$

The nature of the eigenvectors is such that the product $\mathbf{X}_{d}^{H} \mathbf{x}_{i}$ is a column vector with value 1 for entry $i$ and zeros elsewhere. This means the overall product $\mathbf{X}_{d}^{H} \mathbf{x}_{i} \mathbf{x}_{i}^{H} \mathbf{X}_{d}$ has zeros for all entries except entry $i, i$ where it is unity. The overall expression from Eq. (17) thus has zeros for all entries, except for entry $i, i$ where it has a value of $\Delta \lambda_{i} / \lambda_{i}$. Eq. (16) thus simplifies to

$$
\widehat{\mathbf{G}}=\widehat{\mathbf{G}}_{s}+\operatorname{diag}\left(1, \ldots, 1+\Delta \lambda_{i} / \lambda_{i}, \ldots, 1\right) .
$$

Since the eigenvector relationships of Eq. 13 do not change as long as the additive matrix is diagonal, the consequence of Eq. (18) is that for a positive $\Delta \lambda_{i}$ or strengthened background source, the corresponding eigenvalue of $\widehat{\mathbf{G}}$ will be greater than unity and retained by the proposed method when it should be rejected. For a negative $\Delta \lambda_{i}$ or weakened background source the corresponding background eigenvalue of $\widehat{\mathbf{G}}$ will be less than unity and rejected as desired. A source which disappears, where $\Delta \lambda_{i} / \lambda_{i}=-1$, will still be properly rejected by the method. The overall result is that a strengthened background source is at least partially retained by this method, just as it would be by traditional background subtraction. A weakened background source is properly rejected by this method, while depending on the specific source strengths traditional background subtraction could yield negative powers. The method has the potential to have superior performance when compared with traditional background subtraction, at least when the assumption of orthogonal aeroacoustic and background source subspaces holds.

\section{Validation with Simulated Signals}

As a first step of validation, the method must be applied to a problem with a known solution and compared in behavior to traditional background subtraction. This is best done with an analytic acoustic source for which the exact solution is precisely known. This single-source solution will yield a source CSM with one non-zero eigenvalue. To somewhat stress both the traditional and eigenvalue-based methods, a real background acoustic measurement from a wind tunnel test is used rather than simulating background sources. 
The background noise data are taken from the recent Hybrid Wing Body N2A-EXTE aeroacoustic test in NASA Langley's 14- by 22-Foot Subsonic Tunnel 18 A photograph of the test with the model installed in the facility is shown in Fig. 1. This shows the relative position of the array and tower microphones relative to the facility test section for a typical model configuration. For the considered cases, empty tunnel data are used with the phased array center stationed 197 inches downstream from the test section inlet. Mach numbers of $M=0.11,0.17$, and 0.23 are considered. Array microphones with questionable behavior are removed from the CSM prior to processing so as not to affect the eigenvalue analysis, leaving 89 of the original 97 microphones.

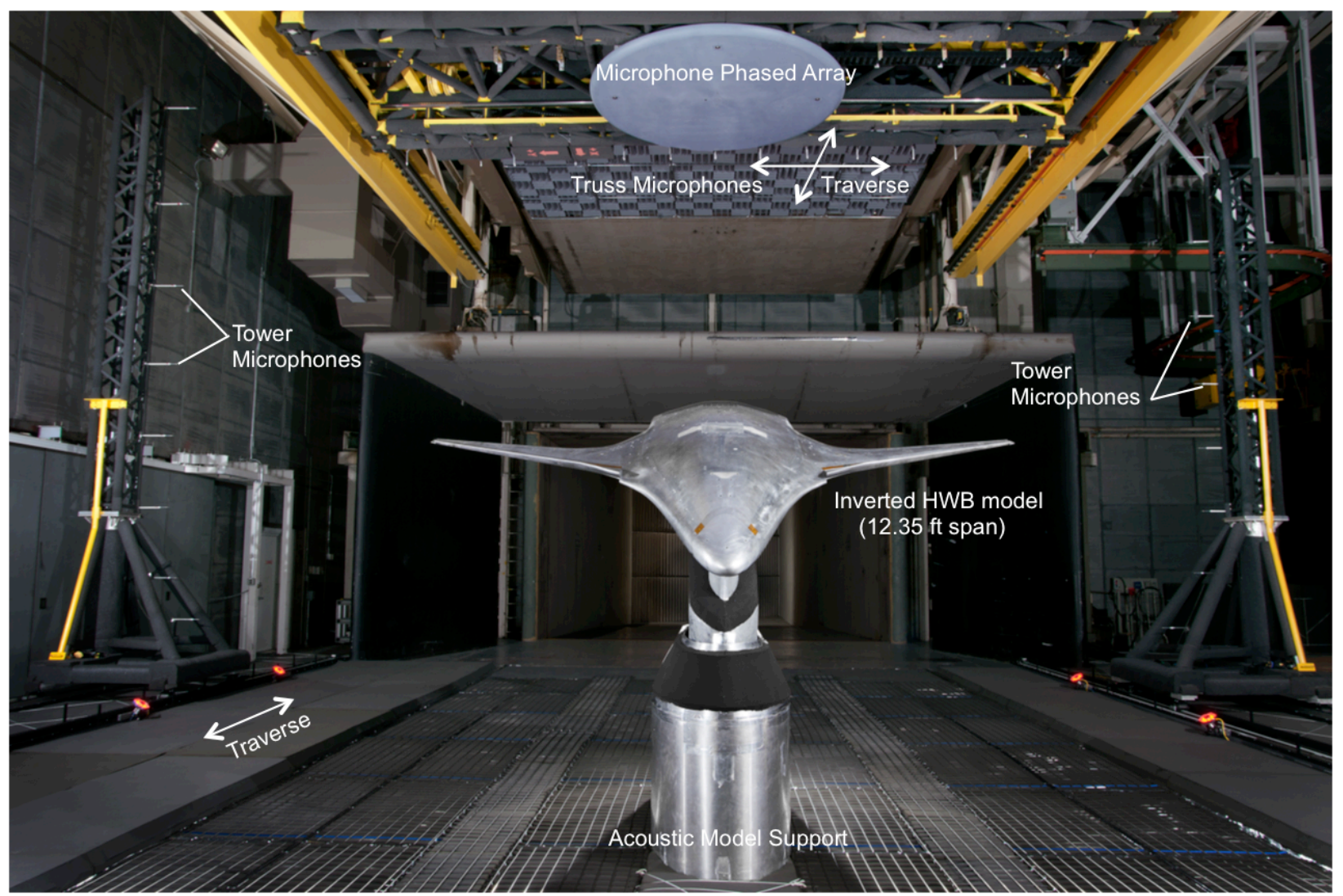

Figure 1. Example arrangement of the HWB model, phased array and tower traverses.

For ease of study, a plane wave propagating in quiescent free space is selected as the acoustic source of interest. Atmospheric attenuation and microphone directivity effects are not modeled. The plane wave

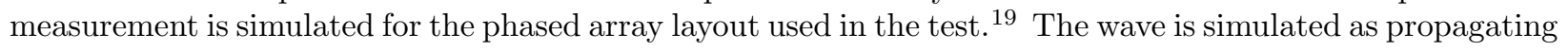
normal to the flow direction and parallel to the array face at the speed of sound associated with the $M=0.17$ background data, and added to the $M=0.17$ background data. The plane wave is defined to have a level of $40 \mathrm{~dB}$ using the pre-selected $30.5 \mathrm{~Hz}$ binwidths of the default data analysis from the test over a frequency range of $4 \mathrm{kHz}$ to $16 \mathrm{kHz}$, and no power at other frequencies. This gives an acoustic source level slightly more than $11 \mathrm{~dB}$ below the facility background level observed by the array center microphone at $4 \mathrm{kHz}$, and slightly more than $4 \mathrm{~dB}$ above the background for the same microphone at $16 \mathrm{kHz}$. Analysis is limited to the $2 \mathrm{kHz}$ to $20 \mathrm{kHz}$ decade. Five different cases are considered:

1. The acoustic source summed with the $M=0.17$ background data are processed with the $M=0.17$ background data. Traditional background subtraction will, within floating point accuracy, identically recover the acoustic signal for this situation.

2. The acoustic source summed with the $M=0.17$ background data are processed with the $M=0.17$ background data, but the isolated background data are artificially decreased by $3 \mathrm{~dB}$ prior to analysis. This simulates a gain in background levels from the background acquisition to the acoustic source acquisition. Traditional background subtraction will overpredict acoustic source levels. 
3. The acoustic source summed with the $M=0.17$ background data are processed with the $M=0.17$ background data, but the isolated background data are artificially increased by $3 \mathrm{~dB}$ prior to analysis. This simulates a reduction in background levels from the background acquisition to the acoustic source acquisition. Traditional background subtraction will either underpredict acoustic source levels or yield negative powers.

4. The acoustic source summed with the $M=0.17$ background data are processed using the $M=0.11$ background data. This simulates a poor estimate of the background data which is lower in level than the true background.

5. The acoustic source summed with the $M=0.17$ background data are processed using the $M=0.23$ background data. This simulates a poor estimate of the background data which is higher in level than the true background.

Note that all CSM data from the experiments are stored as single precision. These are re-cast to double precision for the intermediate calculations and matrix inversions to minimize potential issues with small eigenvalues (though none were observed with single precision calculations). While only 1 decade of the data are shown, the process is conducted on all 4096 bins of the CSMs. Run times are significantly longer than conventional background subtraction, but still approximately 25 seconds per case with $89 \times 89 \times 4096$ CSM data arrays using a MATLAB 2015a implementation on a Macbook Pro with 16 GB of RAM and an Intel Core i7 4960HQ processor.

The results for the array center microphone are shown in Fig. 2. The signals from the simulated source of interest, both clean and with real background signal, are shown in Fig. 2a. This plot shows how the source of interest is fully masked by background noise at lower frequencies and visible at higher frequencies. The results for case 1 shown in Fig. 2b demonstrate that for a correct background measurement both methods recover the source of interest's signal. Fig. 2c shows that when the background acquisition measures a lower background level than that present with the source of interest, both methods fail to recover the correct spectrum. Fig. 2d shows that when the background acquisition measures a higher background level than that present with the source of interest, conventional background subtraction fails, often predicting negative powers (set to negative infinity on the $\mathrm{dB}$ scale for plotting). Conversely, the eigenvalue-based method correctly reconstructs the signal from the source of interest. Fig. 2e shows that when the background acquisition is for a lower Mach number free stream with lower background levels, both methods fail to recover the correct spectrum. Fig. 2f shows that conventional background subtraction fails for a higher Mach number free stream, predicting negative powers for all frequencies plotted. Eigenvalue-based subtraction predicts a correct spectrum within the band of interest, although the spectrum appears noise-contaminated at frequencies where no signal is present. The overall picture of autospectral behavior is that the eigenvalue method never performs worse than conventional background subtraction, and performs better in some situations where conventional subtraction fails.

The results for an example cross-spectral magnitude from the process are shown in Fig. 3 . This shows the cross-spectral magnitude between microphones 1 (the array center) and 10 in the array, where microphone 10 is located 0.46 inches upstream and 1.14 inches laterally from microphone 1 . The overall behavior is similar to that shown for the array center microphone autospectrum. The exact subtraction of case 1 shown in Fig. 3b matches the expected signal in Fig. 3a. The situations where the background acquisition is of lower level than the background of the signal data, either by manual adjustment with case 2 in Fig. 3c or using a lower Mach number background with case 4 in Fig. 3e, show that both methods yield near-identical erroneous results. The situations where the background acquisition is of higher level than the background of the signal data, either by manual adjustment with case 3 in Fig. 3d or using a higher Mach number background with case 5 in Fig. 3f], show that the eigenvalue subtraction correctly predicts the behavior of the signal from the source of interest while conventional subtraction fails to extract the signal of interest from background contamination. As with the autospectral data, eigenvalue subtraction never performs worse than conventional subtraction, and succeeds in some situations where conventional subtraction fails.

The corresponding cross-spectral phase behavior is shown in Fig. 4. Here, the eigenvalue method does show slightly erroneous phase behavior for the case 1 exact subtraction in Fig. $4 \mathrm{~b}$ when compared to the exact solution of Fig. 4a. However, the power at these levels predicted by the method is near-zero in Fig $3 \mathrm{~b}$, so this numerical noise in the reconstruction would likely have minimal effect on any data processing routine. As with previous results, the phase recovery fails equally near the lower band edge for the lower background acquisitions of cases 2 and 4, shown in Fig. 4c and Fig. 4e. The eigenvalue subtraction succeeds 


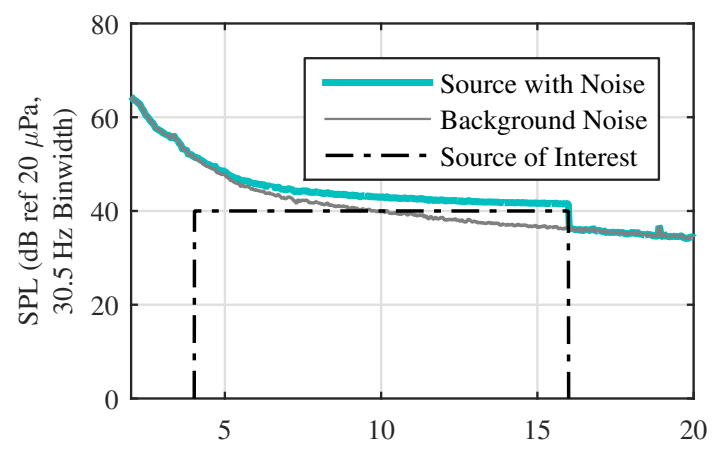

(a) Initial simulated data

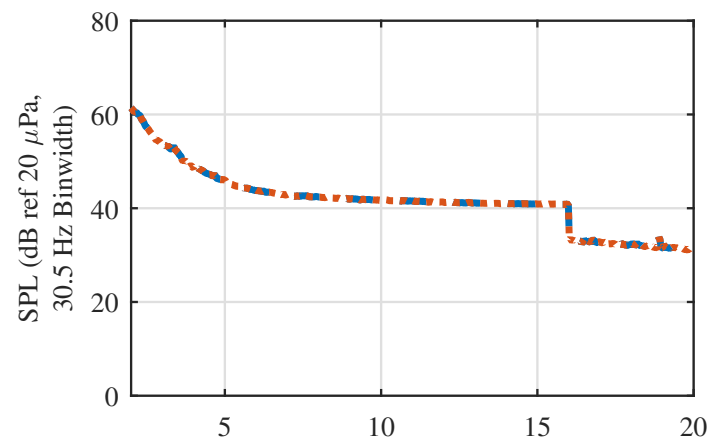

(c) Case 2

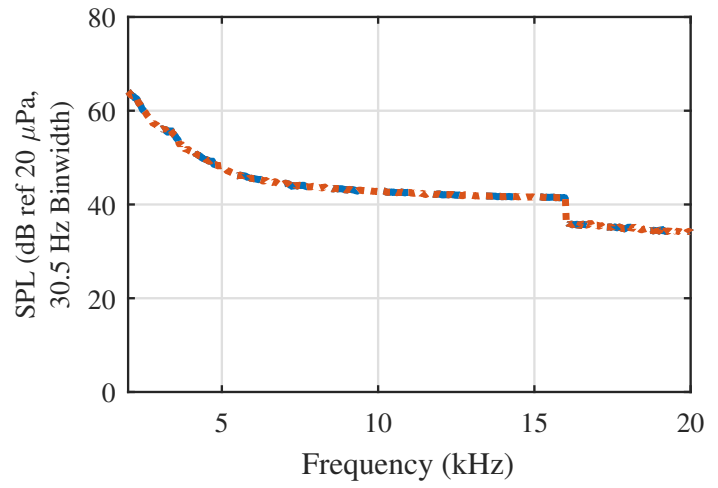

(e) Case 4

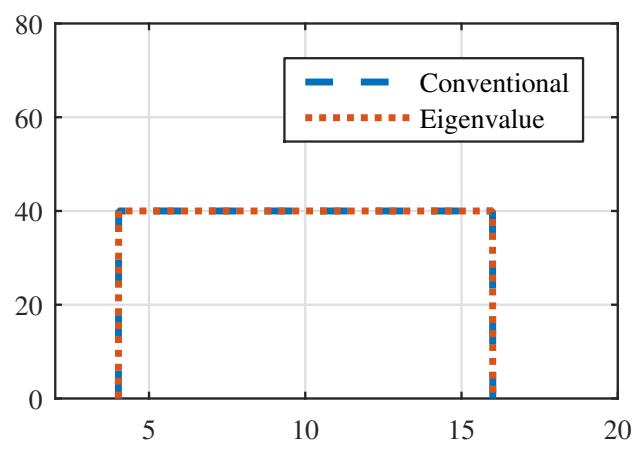

(b) Case 1

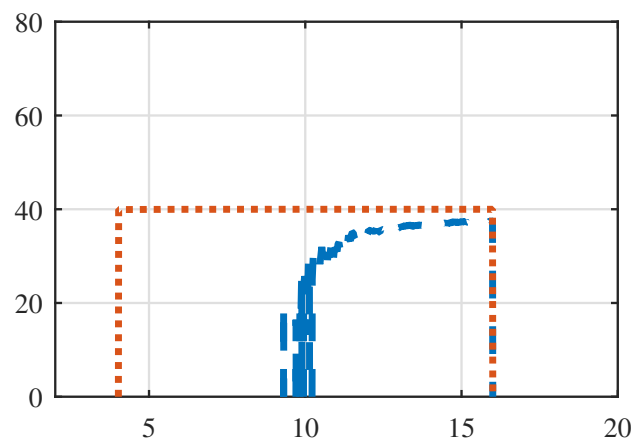

(d) Case 3

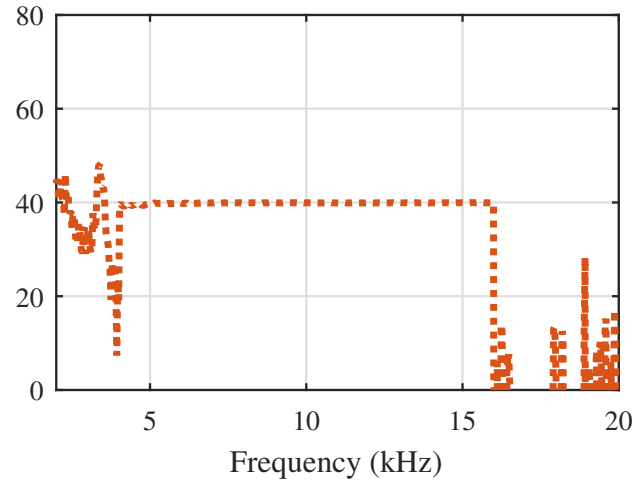

(f) Case 5

Figure 2. Case study of background subtraction methods on the array center microphone autospectrum. 


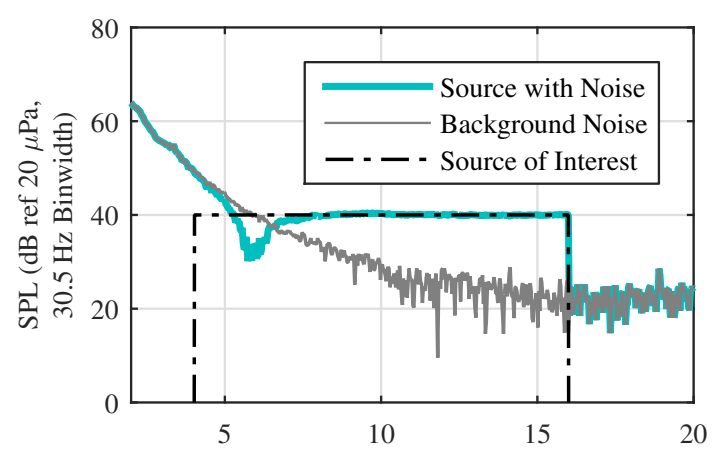

(a) Initial simulated data

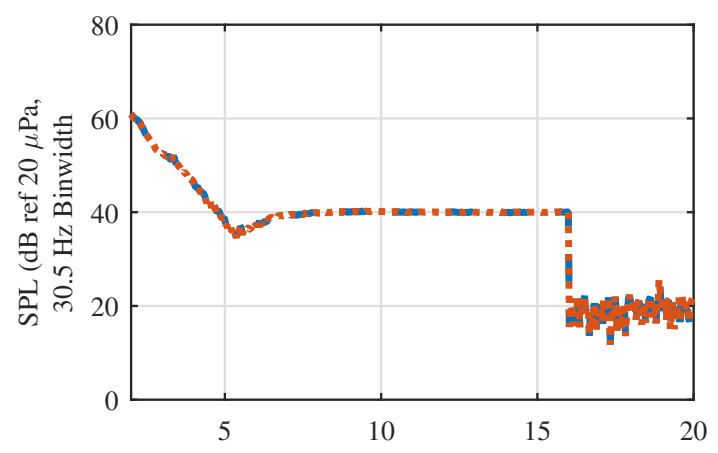

(c) Case 2

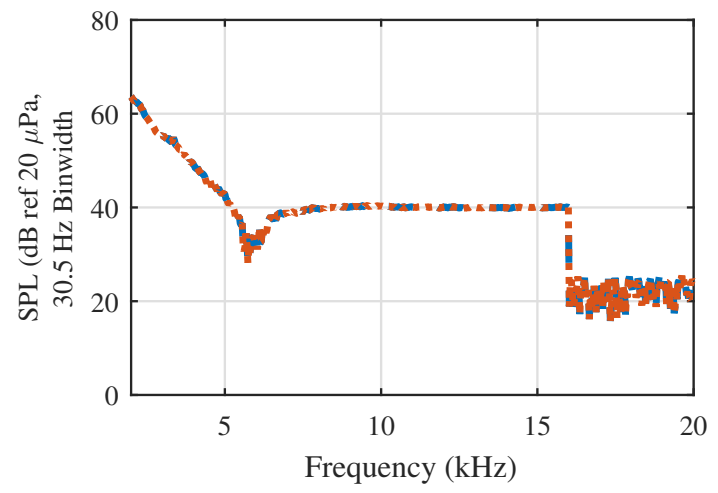

(e) Case 4

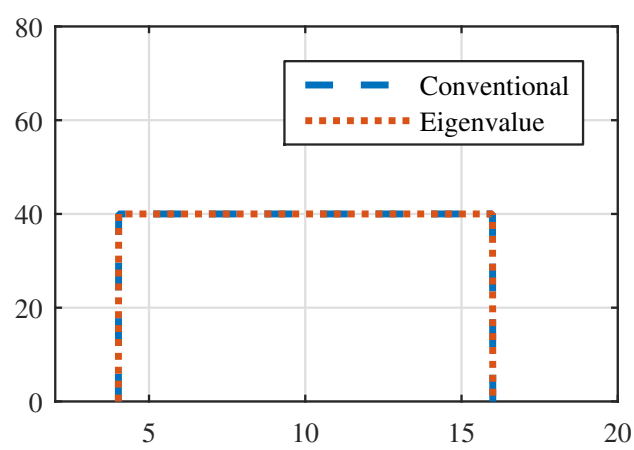

(b) Case 1

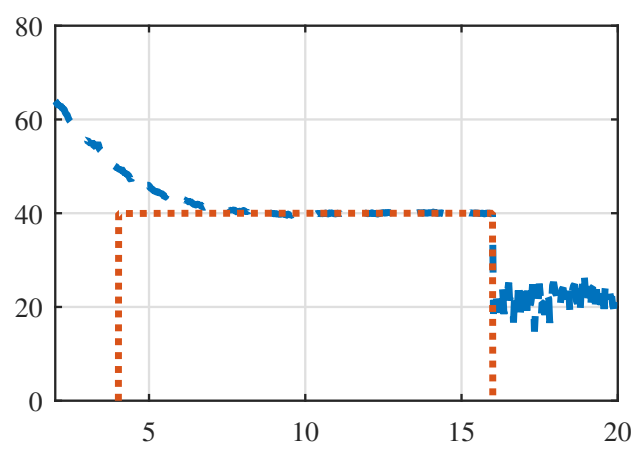

(d) Case 3

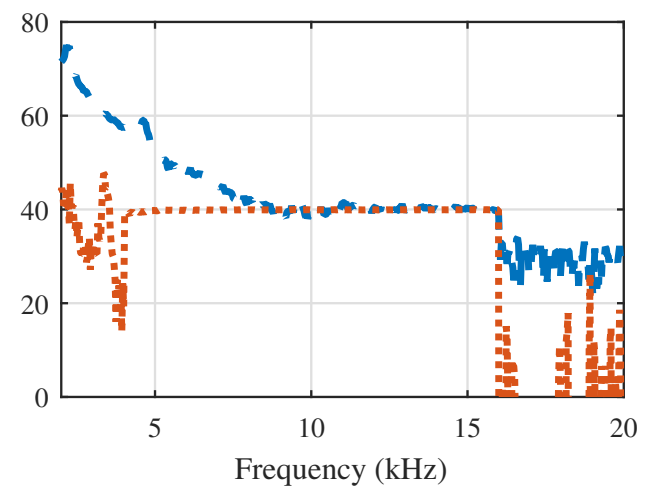

(f) Case 5

Figure 3. Case study of background subtraction methods on the cross-spectral magnitude between the array center microphone and an array microphone located .46 inches upstream and 1.14 inches laterally from the array center. 
in the high background acquisitions of cases 3 and 5 in Fig. 4d and Fig. 4f. while conventional subtraction fails, at least in the poor signal-to-noise band of $4 \mathrm{kHz}$ to $6 \mathrm{kHz}$. As with autospectra and cross-spectral magnitudes, eigenvalue subtraction appears to show superior performance in recovering phase in low signalto-noise measurements. The only time it performs worse than conventional background subtraction is in situations where the cross-spectral magnitude is near-zero power and the exact correct background levels are used. Thus, the eigenvalue subtraction method could prove highly beneficial for any phase-based data analysis.

\section{Evaluation of Experimental Data}

The performance evaluation of background subtraction continues with the use of experimental data. The data analyzed here come from a test campaign in the Arnold Engineering Development Center 40- by 80Foot Wind Tunnel at NASA Ames Research Center, ${ }^{20}$ as well as the aforementioned test in NASA Langley Research Center's 14- by 22-Foot Subsonic Tunnel. While cross-spectral phase was shown in the simulated signal analysis, it is neglected subsequently. Due to the more complicated nature of the signals, plots of phase were found to differ between methods but did not contribute to the presentation of the data.

\section{A. In-flow array}

The first test considered is that using an in-flow array in the 40- by 80-Foot Wind Tunnel. Data were acquired for an in-flow speaker system associated with the AMELIA CESTOL test. Acoustic data were recorded using an OptiNav Array 48 pattern and an in-house data acquisition system. CSMs were generated using the OptiNav Beamform Interactive software package, although the background subtraction used here is performed outside of the OptiNav tool chain. For the data considered here, the facility was operated at a test section Mach number of $M=0.2$. Two different facility drive settings can be used at this Mach number. Data were acquired with the lower-noise of the two. The in-flow speaker system was installed in the test section and given a pink noise input signal at varying input voltage levels. The microphones in this setup experience some degree of self-noise from being exposed to the flow, though they were located outside of the tunnel wall boundary layer. Much of the background noise CSM is hydrodynamic self noise.

Baseline autospectra from array microphone 1 are plotted in Fig. $5 \mathrm{a}$ for speaker input levels of $2.8 \mathrm{~V}$ and $9.0 \mathrm{~V}$, as well as the background level for $0.0 \mathrm{~V}$. The $2.8 \mathrm{~V}$ signal is treated as the signal of interest here as it has a low signal-to-noise ratio for much of the considered bandwidth and warrants application of background subtraction. The $9.0 \mathrm{~V}$ signal is plotted as reference for the expected shape of the speaker spectrum with flow. The $2.8 \mathrm{~V}$ signal without flow is shown as a reference for the overall signal bandwidth and level, although flow-related influence on facility reverberance, propagation, and speaker radiation make this comparison qualitative. The $2.8 \mathrm{~V}$ signal (with flow) becomes nearly indiscernible from the background signal near $14 \mathrm{kHz}$, while features of the $9.0 \mathrm{~V}$ signal are observable up to $18 \mathrm{kHz}$. The cross-spectra between microphones 1 and 2 of the array for the same conditions are plotted in Fig. 5b. The cross-spectrum for the $2.8 \mathrm{~V}$ signal is clearly separate from the background noise up to near $16 \mathrm{kHz}$, while it is distinct for the entire plotted bandwidth of the $9.0 \mathrm{~V}$ signal.

Results of background subtraction are shown in Figs. 5c and 5d. For both auto- and cross-spectra, the conventional and eigenvalue-based subtraction methods are in agreement for frequency bands where the signal is clearly discernible from the noise. Conventional subtraction appears unreliable above approximately $14 \mathrm{kHz}$ in the autospectrum. Eigenvalue-based subtraction appears to perform somewhat better, trending in shape with the $9.0 \mathrm{~V}$ data and appearing to capture the lobe between $15 \mathrm{kHz}$ and $18 \mathrm{kHz}$. However, it finally appears to trend as an offset version of the facility background noise by $20 \mathrm{kHz}$. The eigenvalue-based subtraction shows a more smooth, consistent behavior in bands where the background noise is dominant. For the cross-spectra, both methods are in agreement until nearly $16 \mathrm{kHz}$. The eigenvalue method appears to capture the lobe in the speaker output cross-spectral shape from $15 \mathrm{kHz}$ to $18 \mathrm{kHz}$, albeit with significant fluctuation. The conventional subtraction misses this. Data appear to trend with facility background noise by $20 \mathrm{kHz}$. Aside from the aforementioned lobe, neither method appears to deviate significantly in the magnitude trend from the non-subtracted data in the cross-spectra.

Signal-to-noise ratio estimates are plotted in Fig. 5e, in an attempt to assess an effective noise floor of the subtraction methods for this data set. These are calculated by dividing the results of background subtraction for microphone 1 by the $0.0 \mathrm{~V}$ background data. By its nature, this plot can only show the background-

10 of 21

American Institute of Aeronautics and Astronautics 


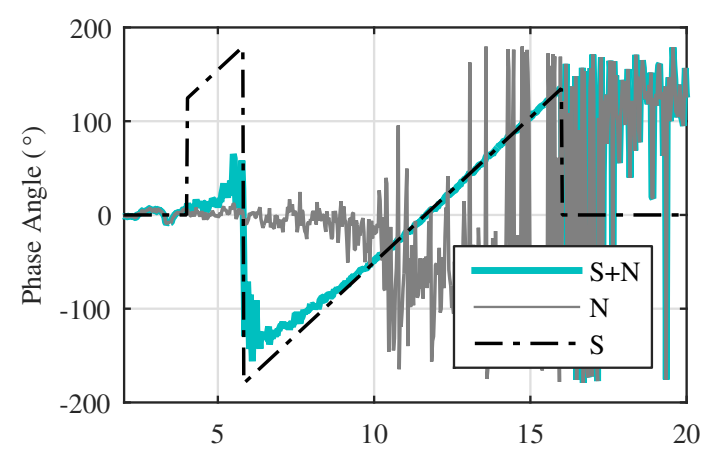

(a) Initial simulated data

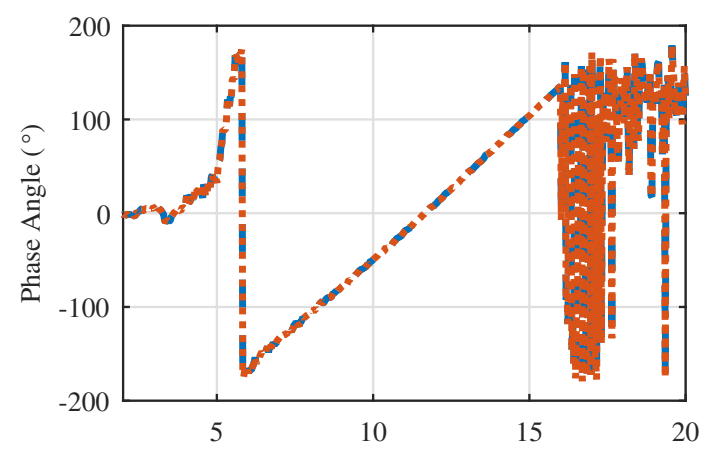

(c) Case 2

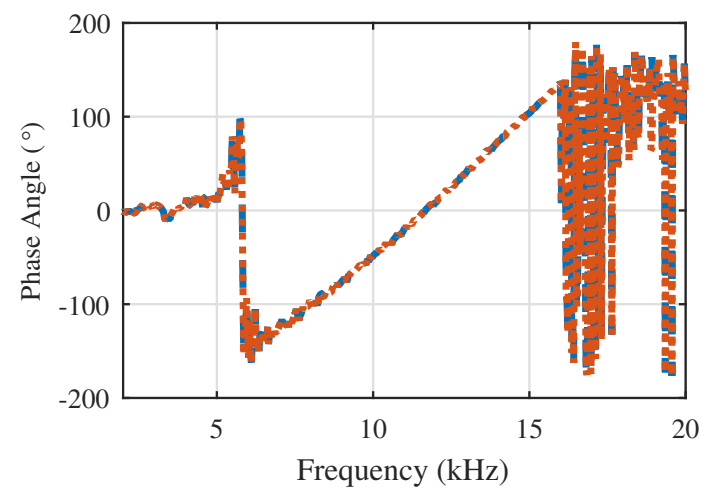

(e) Case 4

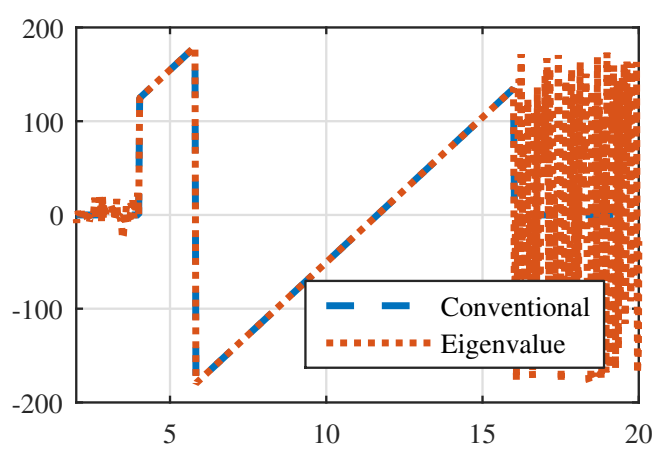

(b) Case 1

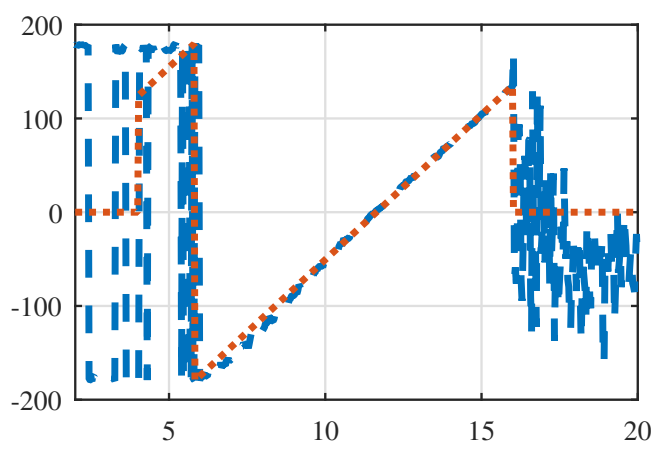

(d) Case 3

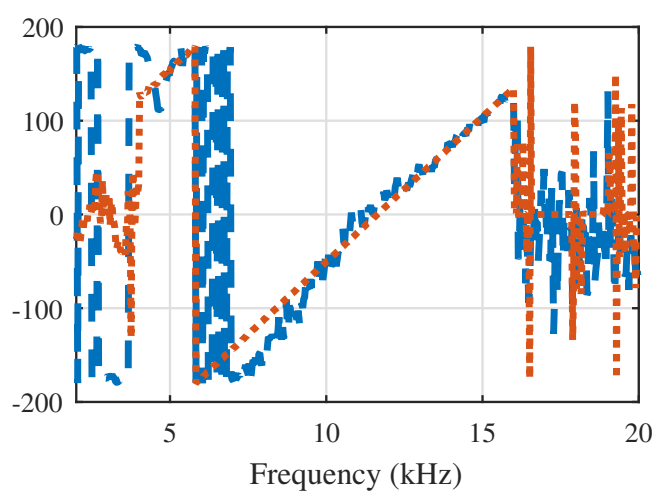

(f) Case 5

Figure 4. Case study of background subtraction methods on the cross-spectral phase between the array center microphone and an array microphone located .46 inches upstream and 1.14 inches laterally from the array center. For plot clarity, the legend in Fig. $4 \mathrm{a}$ has been abbreviated from previous figures. 
subtracted results and thus does not have baseline $2.8 \mathrm{~V}$ and $9.0 \mathrm{~V}$ curves. Note that frequency bins with negative autospectral levels for the microphone are not plotted for conventional background subtraction. Estimates are for the most part similar below $14 \mathrm{kHz}$. They begin to significantly diverge above $14 \mathrm{kHz}$, where both methods drop below ratios of 0.5 . At the higher frequencies the eigenvalue method tends to stay bounded between ratios of 0.1 to 0.3 , while the conventional subtraction is bounded on the upper end by 0.3 and undefined on the lower end with negative estimates. The 0.1 to 0.3 range appears to bound the band where background subtraction can no longer reliably extract information about the signal of interest for the autospectrum.

Coherence-squared $\left(\gamma^{2}\right)$ estimates between microphones 1 and 2 are plotted in Fig. $5 f$ These are shown for the auto- and cross-spectra computed with both subtraction methods as well as those for the baseline 2.8 $\mathrm{V}$ and $9.0 \mathrm{~V}$ signals. Note that frequency bins with negative autospectral levels for either microphone 1 or microphone 2 are not plotted for conventional subtraction. This plot emphasizes one of the main benefits of the eigenvalue subtraction method over conventional background subtraction. Conventional subtraction, as discussed previously, is not constrained to yield a positive semidefinite result of $\mathbf{G}_{s}$. One consequence of this is that the Cauchy-Schwarz inequality is no longer guaranteed to be met by the subtracted data. For this data set at higher frequencies, conventional subtraction yields $\gamma^{2}$ estimates of greater than 100. Conversely, eigenvalue-based subtraction yields a well-bounded estimate of $\gamma^{2} \leq 1$. Additionally, the eigenvalue-based subtraction yields an estimate of $\gamma^{2}$ which trends with the stronger, $9.0 \mathrm{~V}$ signal as opposed to the $2.8 \mathrm{~V}$ signal. This may not have much meaning above $18 \mathrm{kHz}$ where the $9.0 \mathrm{~V}$ signal is also dominated by background noise in its autospectra. It does lend some confidence to the overall trend of the method between $15 \mathrm{kHz}$ and $18 \mathrm{kHz}$, even if individual frequency bins tend to vary noticeably from the $9.0 \mathrm{~V}$ data.

The influence of background subtraction on conventional beamforming for this data is shown in Fig. 6 . Here, one-third octave band spectra of the peak of conventional beamforming output are plotted for both subtraction methods. The beamforming is conducted using the OptiNav Beamform Interactive software package, using most default settings and corrections for 40- by 80 -Foot acoustic data processing. Diagonal removal is applied in the beamforming process.

As shown, background subtraction choice has little influence on the one-third octave band spectral levels from the conventional beamforming operation, even for frequencies where auto- and cross-spectra of the data set may show significant difference between methods. This may be due to summing the data over a large number of narrowband frequencies averaging out disagreement between the two techniques. However, subsequent out-of-flow results demonstrate that isolated narrowband analysis is also similar between the techniques for the selected data, so further investigation is required. Some disagreement is seen at much lower frequencies (not plotted), but at these frequencies neither method appears to be resolving the in-flow speaker. Within the plot data range, the largest disagreement is at $20 \mathrm{kHz}$. Here the conventional method yields a peak of $56.3 \mathrm{~dB}$ while the eigenvalue method yields a peak of $55.7 \mathrm{~dB}$.

Beam maps of the one-third octave band data at $20 \mathrm{kHz}$ are shown for the conventional method in Fig. $7 \mathrm{a}$ and the eigenvalue method in Fig. 7b. The speaker appears in the expected location for this case based on the installation configuration (note flow is from the top of each figure to the bottom), as the peak in the beam map. The beam maps are generated as hemisphere plots covering the whole wind tunnel test section. [4 No photographs were readily-available for superposition with the beam maps. Both beam maps are substantially the same, with minor differences in sidelobe behavior. For this type of source and flow field, choice of subtraction technique appears to have little influence on major features of the beamforming process as long as the source of interest is visible.

\section{B. Out-of-flow array}

The 14- by 22-Foot Subsonic Tunnel Hybrid Wing Body N2A-EXTE test used for the simulated signal analysis is now revisited, but with the model installed in the facility. While the model was built with embedded speakers $\frac{18}{18}$ when these were used they were operated with sufficient power such that most background subtraction analysis would be trivial for the frequency range considered in this work. Instead, an alternate application of background subtraction is considered. During the airframe noise component of the test, one of the configurations considered involved the installation of a drooped leading edge on the model, which generated noise from its exposed side-edge. The following analysis assesses the ability of background subtraction to isolate drooped leading edge noise from other noise sources by subtracting the straight leading edge configuration. Much of the background noise CSM is acoustic noise.

Fig. 8a shows the autospectra of the array center microphone for the drooped and straight leading edge

12 of 21

American Institute of Aeronautics and Astronautics 


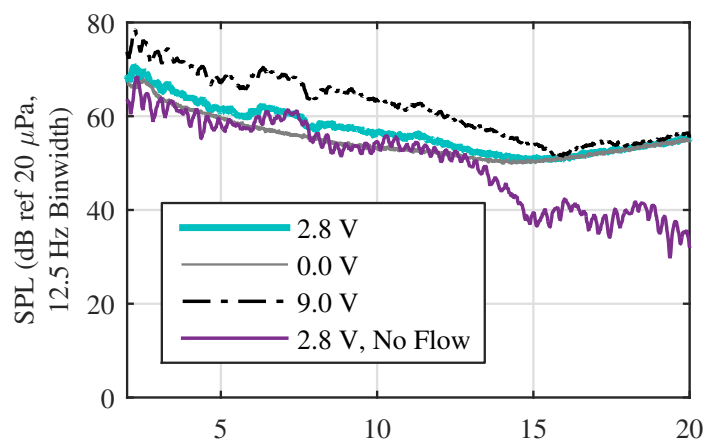

(a) Mic 1 autospectra

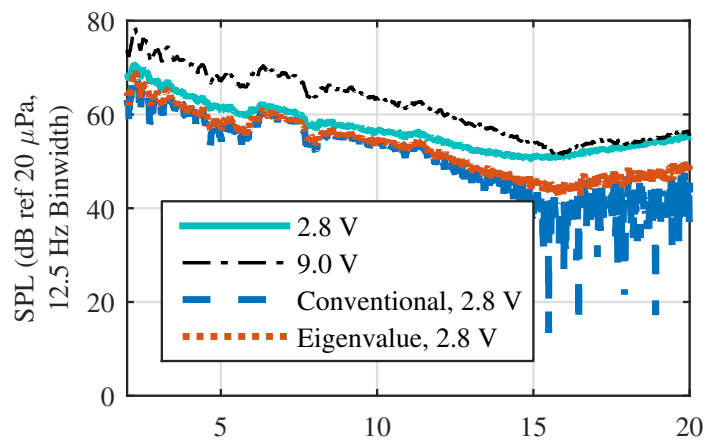

(c) Mic 1 autospectra subtraction

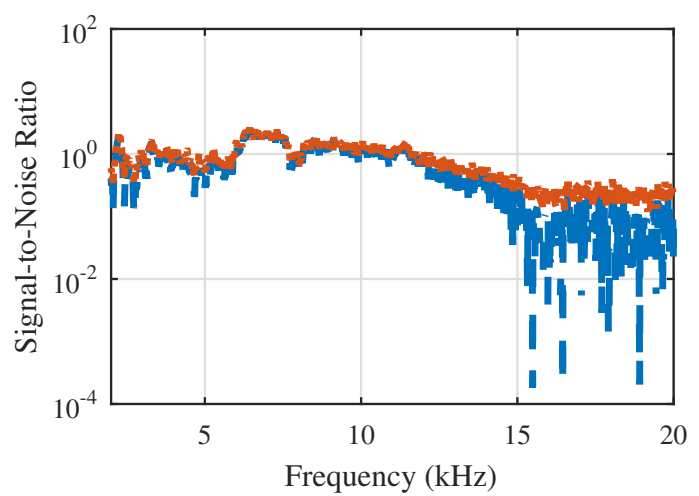

(e) Mic 1 signal-to-noise ratio

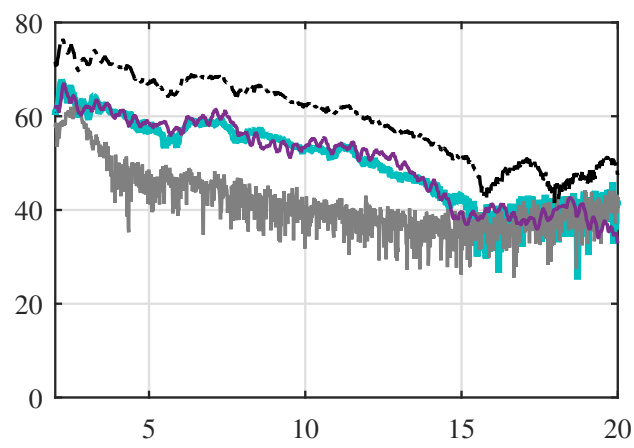

(b) Cross-spectra between mics 1 \& 2

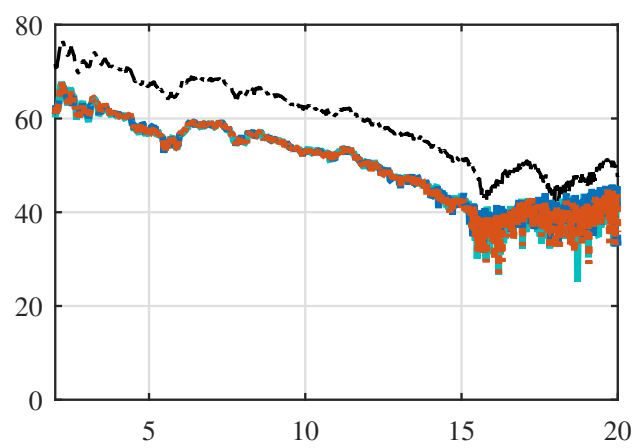

(d) Mic $1 \& 2$ cross-spectra subtraction

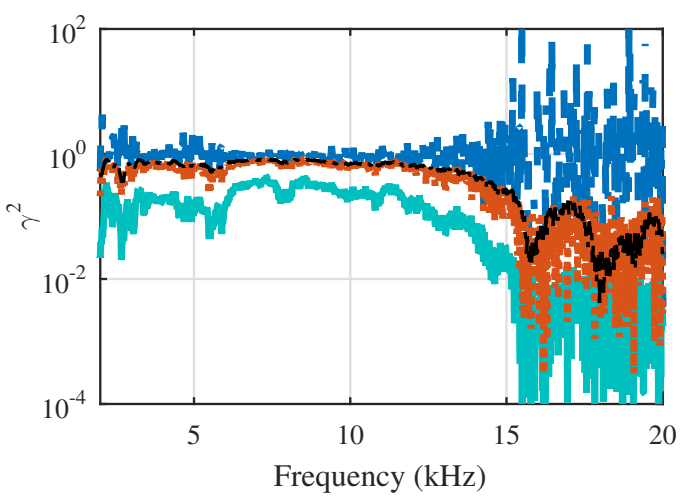

(f) Coherence-squared between mics $1 \& 2$

Figure 5. 40- by 80-Foot Wind Tunnel in-flow Array 48 data from in-flow speaker operation in otherwise empty test section, Mach 0.2 . 


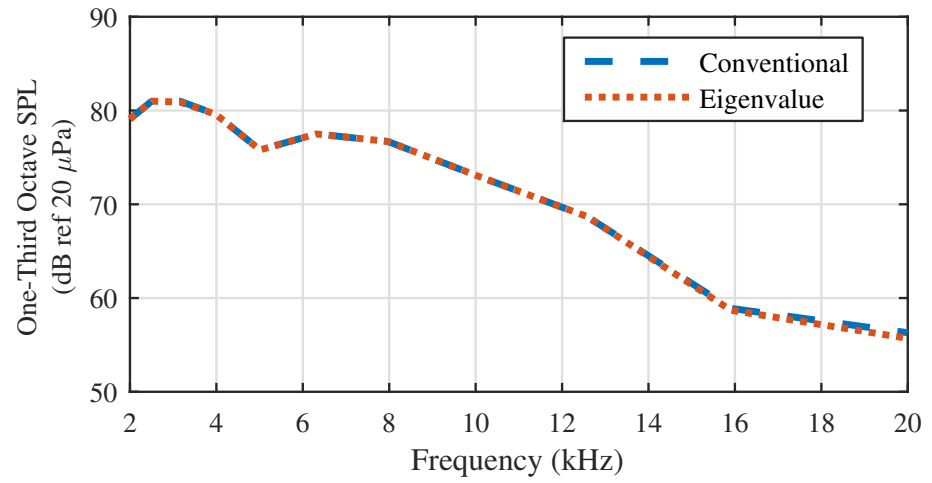

Figure 6. One-third octave band spectra of peak values from conventional beamforming using 40- by 80-Foot in-flow Array 48 in-flow speaker data, Mach 0.20.

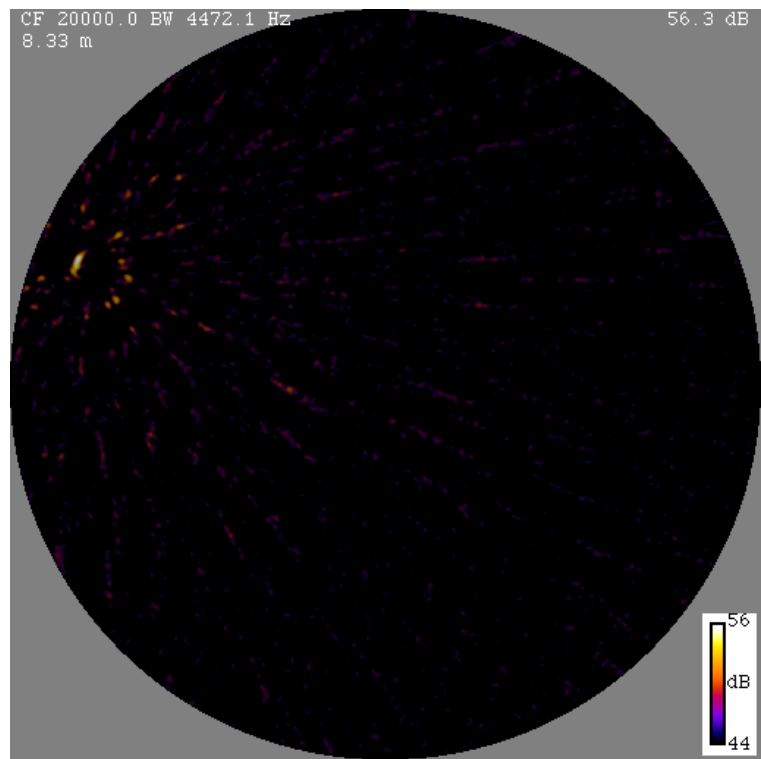

(a) Conventional

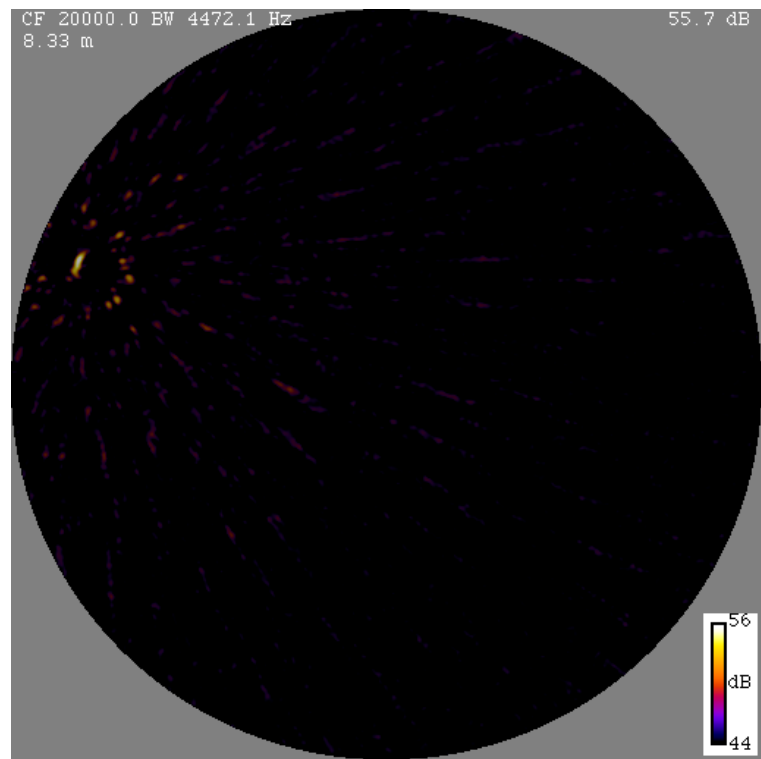

(b) Eigenvalue

Figure 7. $20 \mathrm{kHz}$ one-third octave band conventional beamforming beam maps using 40- by 80-Foot in-flow Array 48 in-flow speaker data, Mach 0.20 (with diagonal removal). 
configurations. For both of these measurements all other airframe components are held constant, the model is installed at a $13.1^{\circ}$ angle of attack, and the test section Mach number is 0.17 . The signal from the drooped leading edge is clearly separable from the straight leading edge configuration above approximately $8 \mathrm{kHz}$. In the cross-spectral magnitudes of Fig. 8b it appears separable above $7 \mathrm{kHz}$, although drops below the background at high frequencies due to the nature of addition in the complex plane.

Background subtraction is applied and plotted in Figs. $8 \mathrm{c}$ and $8 \mathrm{~d}$, under the assumption that the drooped leading edge noise is statistically independent from any of the other dominant noise sources on the airframe. The autospectra from both background subtraction methods are in substantial agreement between $10 \mathrm{kHz}$ and $15 \mathrm{kHz}$. Between $8 \mathrm{kHz}$ and $10 \mathrm{kHz}$ and above $15 \mathrm{kHz}$ conventional subtraction yields results of slightly lower level than the eigenvalue-based subtraction. Below $8 \mathrm{kHz}$ the two results show significant difference in the autospectra. The cross-spectra show closer agreement down to $5 \mathrm{kHz}$, although the conventional method shows more variation in cross-spectral magnitude below $7.5 \mathrm{kHz}$.

Signal-to-noise ratio, computed by treating the straight leading edge configuration as the noise estimate, and coherence estimates in Figs. $8 \mathrm{e}$ and $8 \mathrm{f}$. respectively, show significant divergence in the methods below approximately $8 \mathrm{kHz}$. Both values for the conventional subtraction method break down in this frequency band. The eigenvalue-based subtraction appears to yield consistent results down to $2 \mathrm{kHz}$, although autoand cross-spectral shapes suggest the results may be attenuated acoustic sources from the subtracted acquisition rather than real droop noise. Beamforming results (not shown) indicate that the wingtip vortices are dominant at these lower frequencies even with background subtraction.

Based on the behavior of the data, signal-to-noise and coherence calculations, further analysis now focuses on the $7 \mathrm{kHz}$ narrowband component of the data. This appears to be a frequency where the drooped leading edge noise may be significant and the two subtraction methods yield significantly different results for the center microphone autospectra and conventional subtraction is questionable. This is conducted by performing conventional beamforming and then applying the DAMAS deconvolution algorithm. 21

Conventional beamforming allows for a quick assessment of the noise sources present in the data and the influence of different parameters on the array data processing. For all of the presented beam maps, the beamforming formulation follows that of Eq. 9 in Ref. 21, although the denominator of the expression is changed to

$$
\left(\sum_{m=1}^{m_{0}} w_{m}\right)^{2}-\sum_{m=1}^{m_{0}} w_{m}^{2},
$$

using the original authors' notation for microphone indices and weighting factors. This alteration provides a unit-power-in, unit-power-out relationship for an isolated point source. The frequency-dependent microphone weighting scheme is the same which has been previously-defined for this test $\frac{19}{19}$ Conventional beamforming is conducted with steering vectors accounting for shear layer refraction, atmospheric attenuation and microphone directivity corrections, although the latter two effects have a negligible influence on the data at 7 $\mathrm{kHz} \cdot \stackrel{[2]}{T}$

The data are processed both with and without background subtraction, and with and without diagonal removal. Diagonal removal is varied here because it is a noise contamination mitigation tool. If a background subtraction is fully-successful, diagonal removal should be unnecessary. This data set is by no means the best tool by which to determine the need for diagonal removal, as diagonal removal is primarily directed towards self-noise and not additional acoustic contamination. However, beamforming and deconvolution both with and without diagonal removal can be used to assess the overall influence of this parameter for this type of analysis.

Conventional beamforming results without background subtraction are shown in Fig. 9. These results show that the drooped leading edge noise sources, the hot spots on the inboard section of the hybrid wing body wings, are on the same order of strength as the noise from the wingtip vortices. Diagonal removal visually isolates these dominant noise sources, and shows a noticeable decrease in the peak level of the beam maps. Conventional beamforming results with conventional background subtraction are shown in Fig. 10 Note that application of conventional background subtraction without diagonal removal can be a dangerous prospect. While it appears to yield a sensible beam map in this case, the inclusion of negative autospectral powers in the conventional beamforming equation could yield unpredictable results under different circumstances and is not recommended without additional investigation of the data behavior. Regardless, results show that the subtraction successfully removes the wingtip vortices from the data while introducing significant low-level lobes in the beam maps. The eigenvalue-based subtraction results, shown in Fig. 11, also show successful 


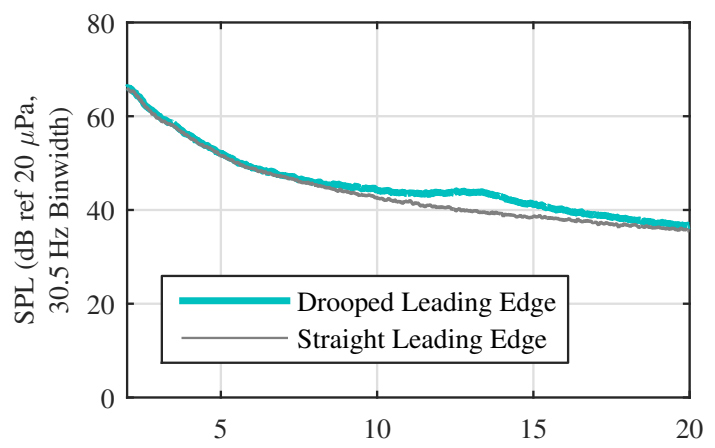

(a) Mic 1 autospectra

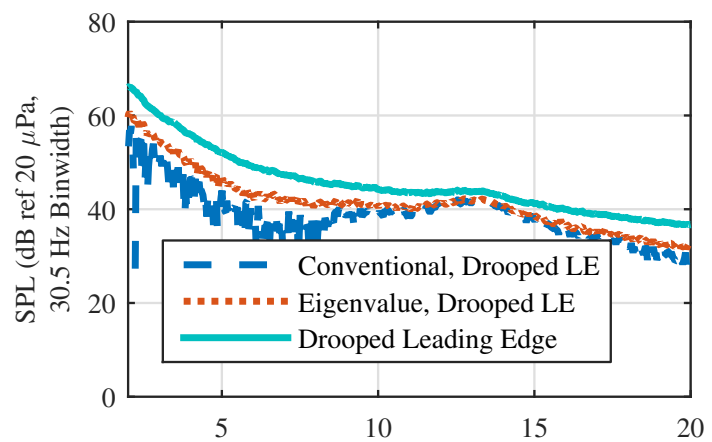

(c) Mic 1 autospectra subtraction

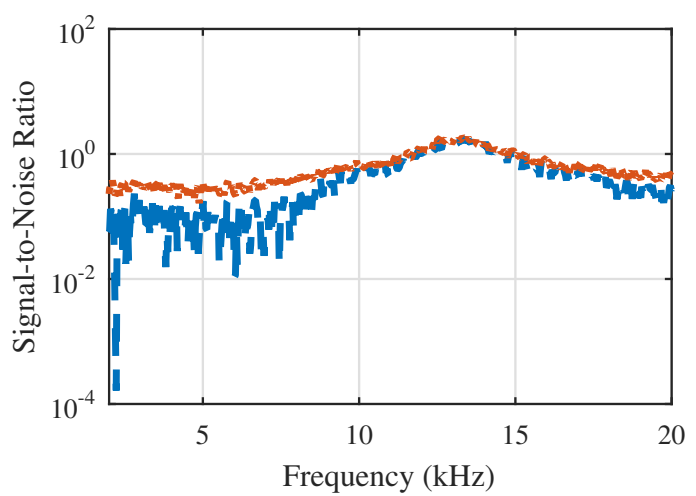

(e) Mic 1 signal-to-noise ratio

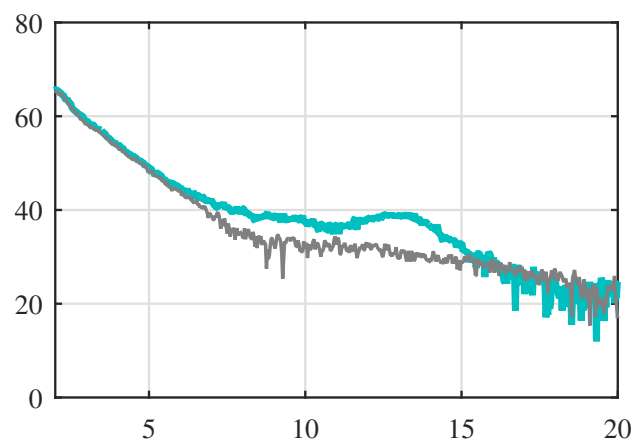

(b) Cross-spectra between mics $1 \& 10$

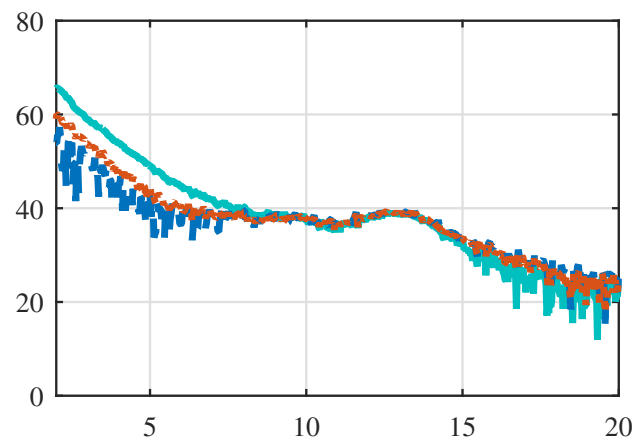

(d) Mic 1 \& 10 cross-spectra subtraction

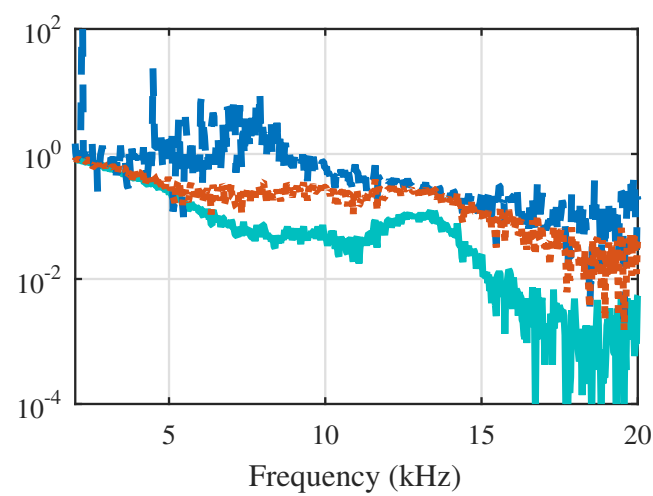

(f) Coherence-squared between mics $1 \& 10$

Figure 8. 14- by 22-Foot Subsonic Wind Tunnel out-of-flow phased array data for leading-edge droop with model angle of attack of $13.1^{\circ}$ and test section Mach number of 0.17 . 
isolation of the drooped leading edge noise sources. The eigenvalue-based method shows a consistent, uniform floor for much of the beam map when diagonal removal is not applied, and reduced additional lobe presence when diagonal removal is applied and results compared to conventional subtraction.

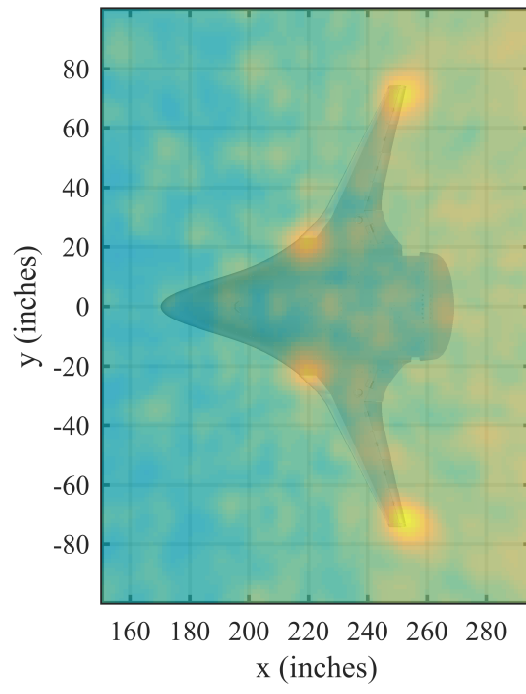

(a) Baseline

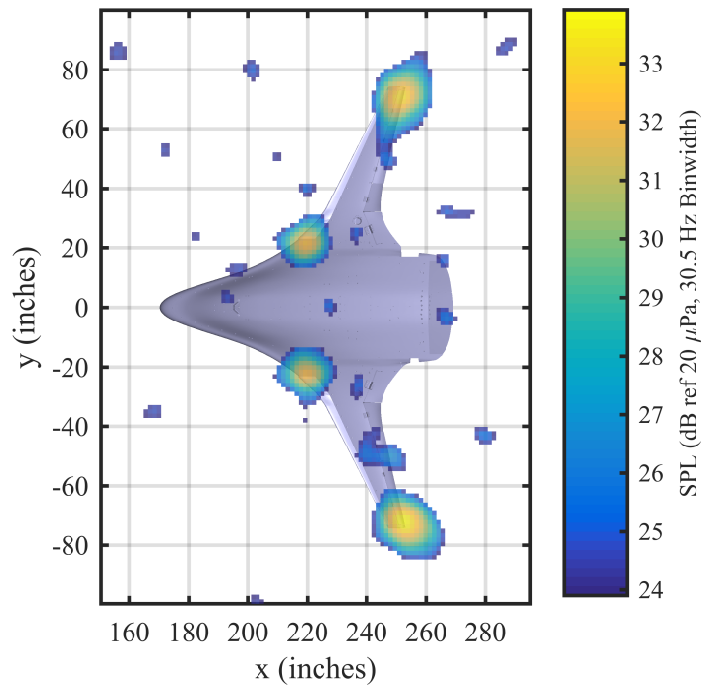

(b) Diagonal removal

Figure 9. HWB drooped leading edge $7 \mathrm{kHz}$ beam maps with no background subtraction.

DAMAS results without background subtraction are shown in Fig. 12 These and subsequent results are shown for 200 Gauss-Seidel passes on the grid, using four different sweep directions to mitigate energy migration through the deconvolution results. Results are now plotted on a $20-\mathrm{dB}$ scale instead of the previous 10-dB scale. Qualitatively, the results separate the wingtip vortices and droop leading edge noise sources for both cases. However, significant deconvolution artifacts are present in the results without diagonal removal, shown in Fig. 12a. The diagonal removal results in Fig. 12b show improved visual behavior.

Table 1. Evaluation metrics for influence of diagonal removal (DR) with varying background subtraction methods.

\begin{tabular}{|c||c|c|c|}
\hline & Integrated Power, No DR & Integrated Power, DR & relative difference norm \\
\hline \hline No Subtraction & $39.5 \mathrm{~dB}$ & $38.8 \mathrm{~dB}$ & 0.753 \\
\hline Conventional & $38.2 \mathrm{~dB}$ & $39.0 \mathrm{~dB}$ & 1.05 \\
\hline Eigenvalue & $38.4 \mathrm{~dB}$ & $38.5 \mathrm{~dB}$ & 0.259 \\
\hline
\end{tabular}

The differences between the two results are assessed quantitatively through two metrics. The first is the integrated power from the drooped leading edges. This is computed by drawing a 20" by 20 " square around each droop side-edge and summing the power contribution from both square regions. The second metric is the Euclidean norm of the difference, in $\mathrm{Pa}^{2}$, between the two deconvolution maps, normalized by the Euclidean norm of the deconvolution map without diagonal removal. Results for all cases are shown in Table 1

DAMAS results with conventional background subtraction are shown in Fig. 13. Significant differences exist between the two deconvolution maps both from a quantitative and qualitative perspective. The integrated levels of the leading edge noise are reasonably close to the integrated level of the leading edge noise without subtraction and with diagonal removal. However, there is a noticeable difference between the two deconvolution maps. This is visible qualitatively through additional artifacts in the diagonal removal plot of Fig. 13b not visible in the subtraction-only plot of Fig. 13a, and quantitatively through the growth in the difference norm from the case without subtraction. 


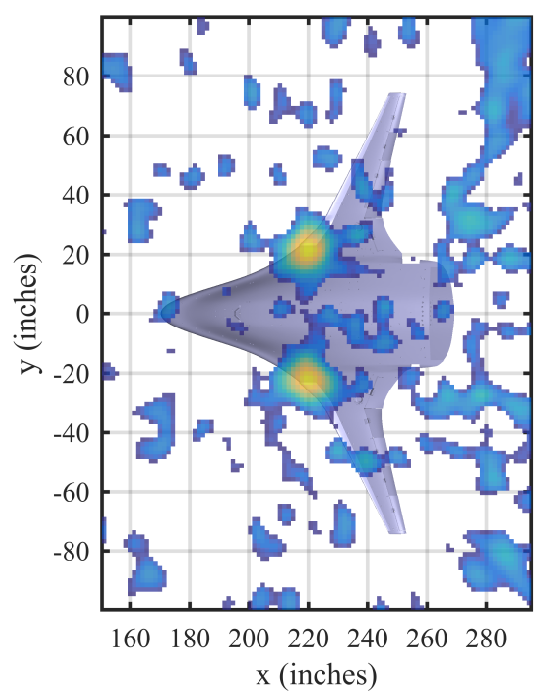

(a) Baseline

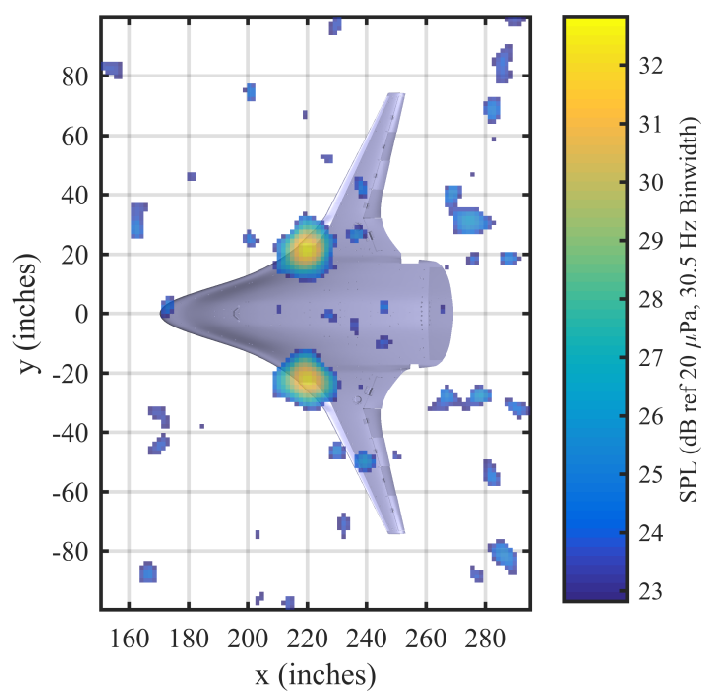

(b) Diagonal removal

Figure 10. HWB drooped leading edge $7 \mathrm{kHz}$ beam maps with conventional background subtraction.

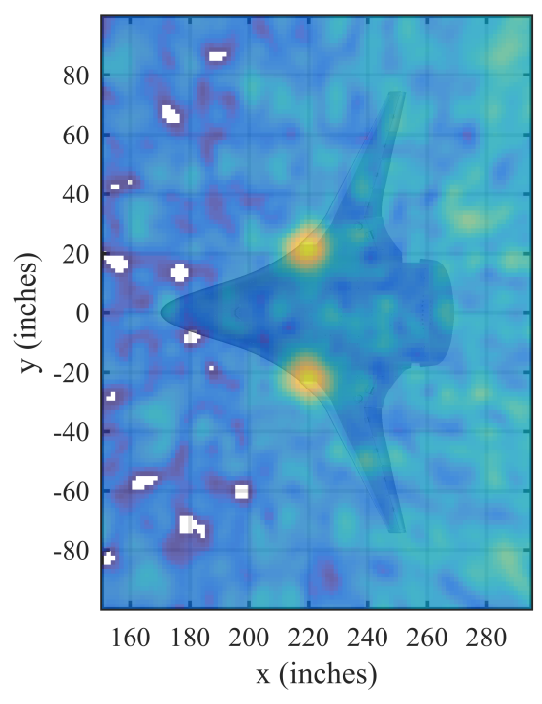

(a) Baseline

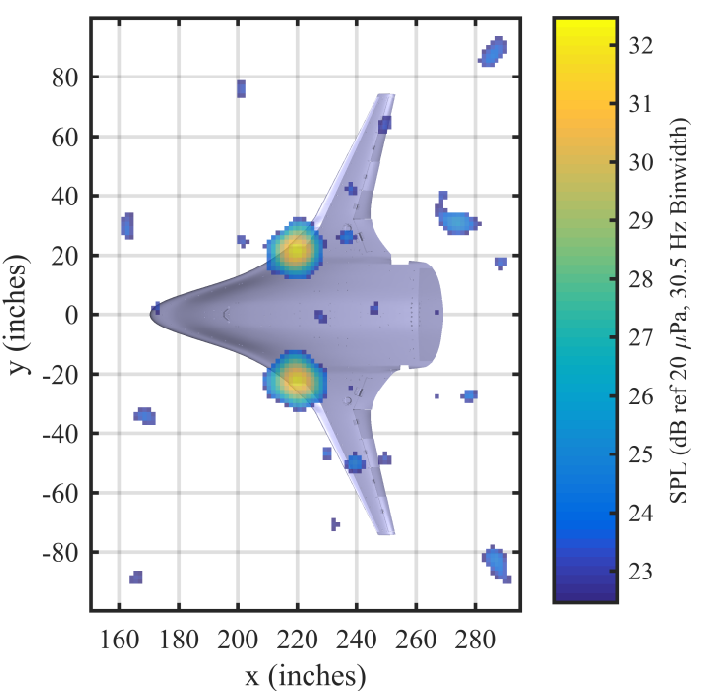

(b) Diagonal removal

Figure 11. HWB drooped leading edge $7 \mathrm{kHz}$ beam maps with eigenvalue-based background subtraction. 
DAMAS results with advanced subtraction are shown in Fig. 14. Qualitatively, these deconvolution maps appear to be the most similar to each other in terms of both source distribution and deconvolution artifacts. This is supported quantitatively by the integrated power calculations being so close, as well as the reduction in the difference norm. While this preliminary analysis does not prove diagonal removal is unnecessary with a successful eigenvalue-based background subtraction, it does build upon the possibility that diagonal removal may not be necessary with a successful eigenvalue-based background subtraction.

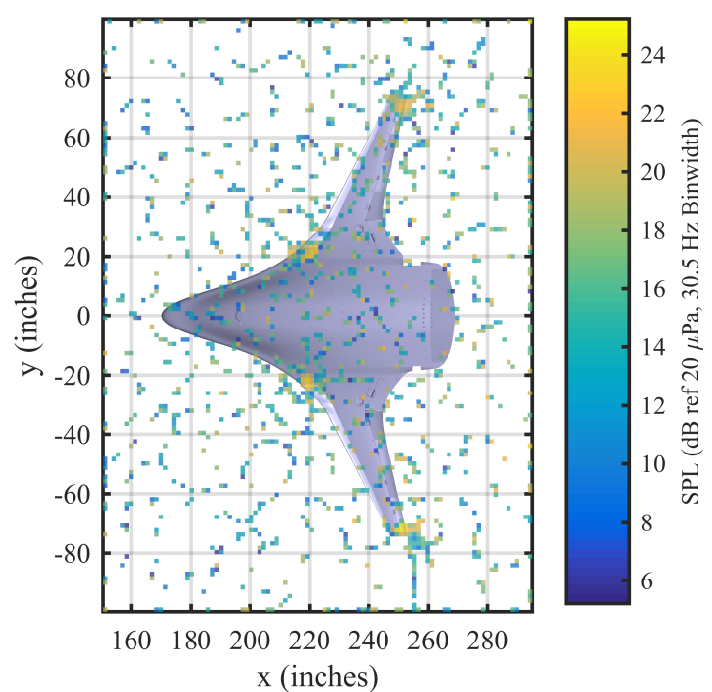

(a) Baseline

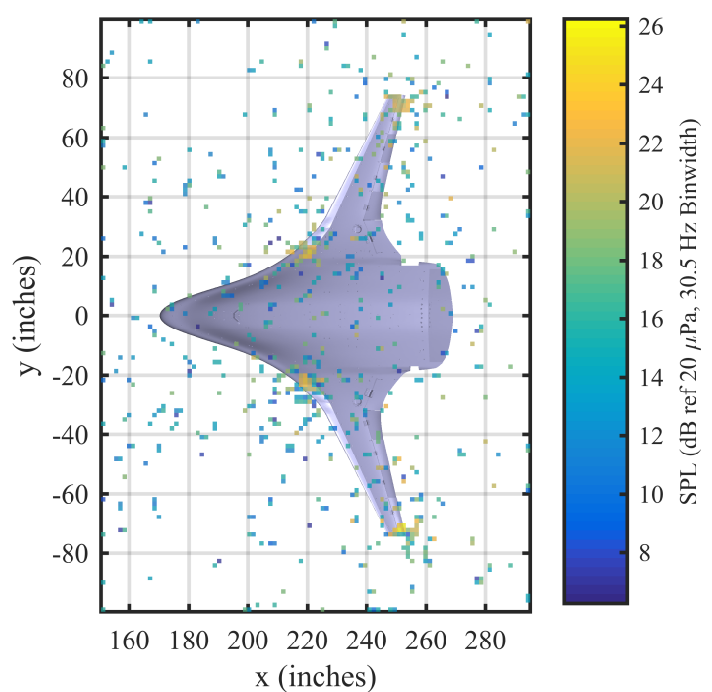

(b) Diagonal removal

Figure 12. HWB drooped leading edge $7 \mathrm{kHz}$ deconvolution maps with no background subtraction.

\section{Summary \& Conclusions}

An advanced form of background subtraction is presented and applied to aeroacoustic wind tunnel data. The background subtraction method identifies eigenvalues in a CSM which are related to background noise sources and removes them, under the assumption that background noise eigenvectors are orthogonal to signal-of-interest eigenvectors.

The eigenvalue-based subtraction technique shows improved, more robust behavior when compared to conventional background subtraction using simulated data. While the technique may still fail under a variety of conditions, it does not appear to fail in a situation where conventional subtraction succeeds. It does succeed in situations where conventional subtraction fails. The technique also provides the benefit of maintaining a positive semi-definite CSM, and physical coherence relationships between channels in the CSM.

Experimental results in a closed-wall wind tunnel data set show some ability to reconstruct an expected spectral shape for data where conventional subtraction cannot. This holds for both auto- and cross-spectra. While the new method still appears to fail for sufficiently weak signal data, it maintains usable signal-tonoise and coherence estimates which allow for assessment of data quality. Conventional beamforming with diagonal removal appears to be insensitive to subtraction method for this type of data set.

Experimental results in an open-jet wind tunnel data set show the technique allows for the separation of two distinct aeroacoustic noise sources, and provides more consistent behavior at frequencies where conventional subtraction fails. The method is applied with conventional beamforming and deconvolution in an attempt to assess whether or not it provides an alternative to diagonal removal. While all considered parameter combinations extract similar source level estimates, the eigenvalue-based subtraction shows the least dependence on the use of diagonal removal in terms of image quality and change in deconvolution maps.

In summary, the proposed background subtraction method allows an alternative background subtraction calculation which, while still limited, is more robust to fluctuations in background noise between tare and signal acquisitions. The method maintains desirable statistical properties of the predicted CSMs. It also 


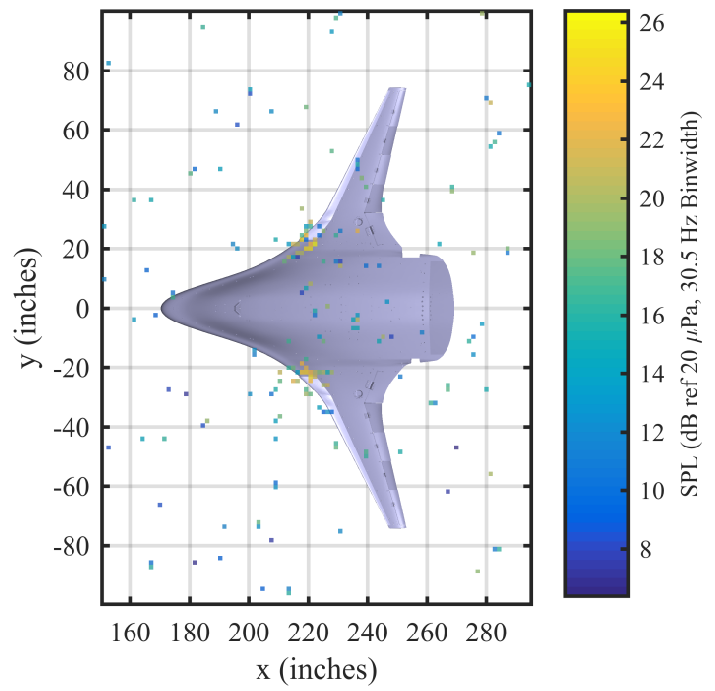

(a) Baseline

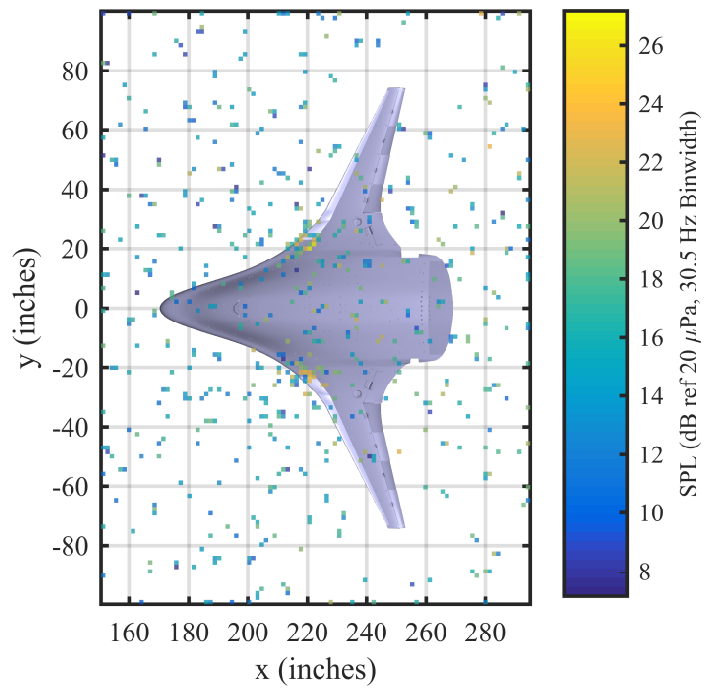

(b) Diagonal removal

Figure 13. HWB drooped leading edge $7 \mathrm{kHz}$ deconvolution maps with conventional background subtraction.

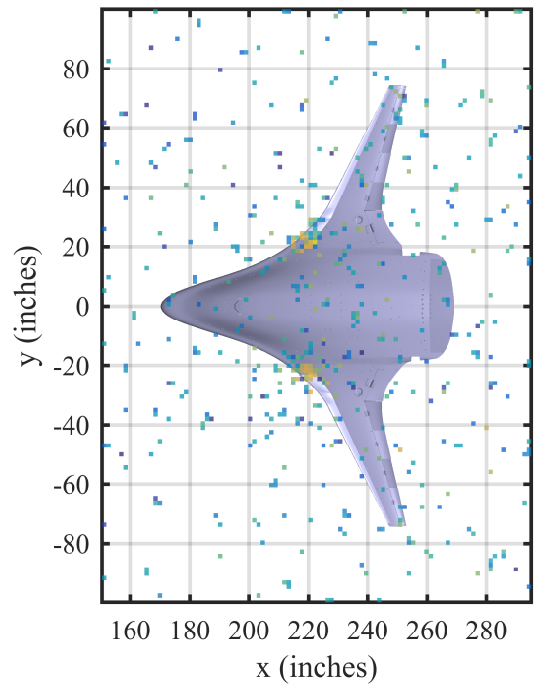

(a) Baseline

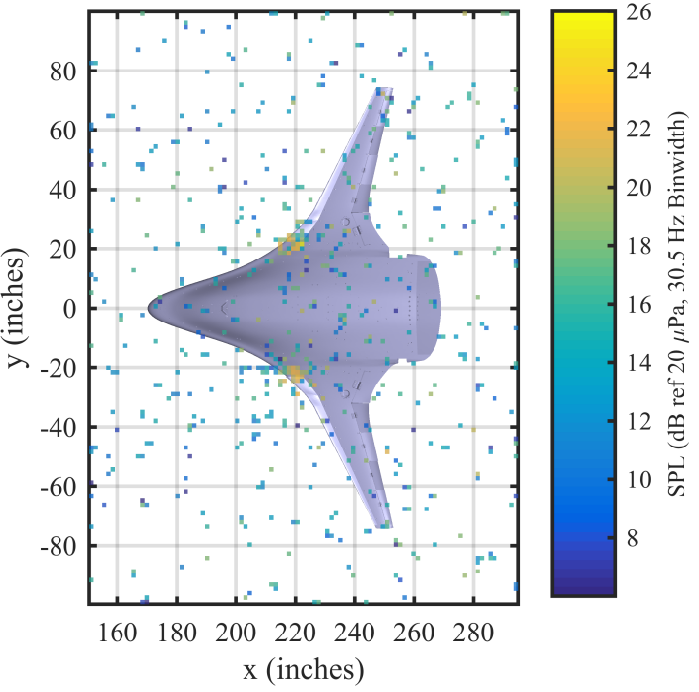

(b) Diagonal removal

Figure 14. HWB drooped leading edge $7 \mathrm{kHz}$ deconvolution maps with eigenvalue-based background subtraction. 
may provide an alternative self-noise mitigation method to diagonal removal when performing beamforming and deconvolution analysis, although further assessment with data tailored to test this is required.

\section{Acknowledgments}

This work was funded by the NASA Environmentally Responsible Aviation and Advanced Air Transport Technology Projects. The authors also wish to acknowledge the efforts of research and test staff at both NASA Langley and NASA Ames for their dedicated work with the N2A-EXTE and AMELIA CESTOL tests.

\section{References}

\footnotetext{
${ }^{1}$ Dougherty, R. P., Aeroacoustic Measurements, T. J. Mueller, Ed., chap. 2. Beamforming in Acoustic Testing, SpringerVerlag, Berlin, Heidelberg \& New York, 2002, pp. 62-97.

${ }^{2}$ Bahr, C. J., Brooks, T. F., Humphreys, W. M., Spalt, T. B., and Stead, D. J., "Acoustic Data Processing and Transient Signal Analysis for the Hybrid Wing Body 14- by 22-Foot Subsonic Wind Tunnel Test," AIAA 2014-2345, 20th AIAA/CEAS Aeroacoustics Conference, AIAA Aviation 2014, Atlanta, GA, June 2014.

${ }^{3}$ Humphreys, W. M., Brooks, T. F., Hunter, W. W., and Meadows, K. R., "Design and Use of Microphone Directional Arrays for Aeroacoustic Measurements," AIAA-98-0471, 36th AIAA Aerospace Sciences Meeting \& Exhibit, Reno, NV, January 1998.

${ }^{4}$ Horne, W. C., "Initial Assessment of Acoustic Source Visibility with a 24-element Microphone Array in the Arnold Engineering Development Center 80- by 120-Foot Wind Tunnel at NASA Ames Research Center," AIAA 2011-2723, 17th AIAA/CEAS Aeroacoustics Conference, Portland, OR, June 2011.

${ }^{5}$ Bahr, C. J., Yardibi, T., Liu, F., and Cattafesta, L. N., "An Analysis of Different Measurement Techniques for Airfoil Trailing Edge Noise," AIAA 2008-2957, 14th AIAA/CEAS Aeroacoustics Conference, Vancouver, BC, May 2008.

${ }^{6}$ Spalt, T. B., Fuller, C. R., Brooks, T. F., and Humphreys, W. M., "A Background Noise Reduction Technique using Adaptive Noise Cancellation for Microphone Arrays," AIAA 2011-2715, 17th AIAA/CEAS Aeroacoustics Conference, Portland, OR, June 2011.

${ }^{7}$ Blacodon, D., "Array Processing for Noisy Data: Application for Open and Closed Wind Tunnels," AIAA Journal, Vol. 49, No. 1, January 2011, pp. 55-66.

${ }^{8}$ Dougherty, R. P., "Source Location with Sparse Acoustic Arrays; Interference Cancellation," DNW, 1st CEAS-ASC Workshop: Wind Tunnel Testing in Aeroacoustics, 5-6 November, 1997.

${ }^{9}$ Oerlemans, S. and Sijtsma, P., "Determination of absolute levels from phased array measurements using spatial source coherence," Nlr-tp-2002-226, NLR, 2002.

${ }^{10}$ Sarradj, E., "A fast signal subspace approach for the determination of absolute levels from phased microphone array measurements," Journal of Sound and Vibration, Vol. 329, No. 9, 2010, pp. 1553-1569.

${ }^{11}$ Suzuki, T., " $L_{1}$ generalized inverse beam-forming algorithm resolving coherent/incoherent, distributed and multipole sources," Journal of Sound and Vibration, Vol. 330, No. 24, 2011, pp. 5835-5851.

${ }^{12}$ Bulté, J., "Acoustic Array Measurements in Aerodynamic Wind Tunnels: a Subspace Approach for Noise Suppression," AIAA 2007-3446, 13th AIAA/CEAS Aeroacoustics Conference, Rome, Italy, 2007.

${ }^{13}$ Allen, M. R. and Smith, L. A., "Optimal filtering in singular spectrum analysis," Physics Letters A, Vol. 234, October 1997, pp. 419-428.

${ }^{14}$ Sekihara, K., Hild, K. E., Dalal, S. S., and Nagarajan, S. S., "Performance of Prewhitening Beamforming in MEG Dual Experimental Conditions," IEEE Transactions on Biomedical Engineering, Vol. 55, No. 3, March 2008, pp. 1112-1121.

${ }^{15}$ Moler, C., Numerical Computing with MATLAB, chap. 10. Eigenvalues and Singular Values, The MathWorks, 2013, pp. $1-39$.

${ }^{16}$ Higham, N. J., "Computing a Nearest Symmetric Positive Semidefinite Matrix," Linear Algebra and its Applications, Vol. 103, 1988, pp. 103-118.

${ }^{17}$ D'Errico, J., "nearestSPD (http://www.mathworks.com/matlabcentral/fileexchange/42885-nearestspd)," (last accessed March 24, 2015), 2013.

${ }^{18}$ Heath, S. L., Brooks, T. F., Hutcheson, F. V., Doty, M. J., Bahr, C. J., Hoad, D., Becker, L. E., Humphreys, W. M., Burley, C. L., Stead, D. J., Pope, D. S., Spalt, T. B., Kuchta, D., Plassman, G. E., and Moen, J., "NASA Hybrid Wing Body Aircraft Aeroacoustic Test Data Report," Tech. Rep. NASA TM (TBD), NASA - Environmentally Responsible Aviation Project, 2015.

${ }^{19}$ Humphreys, W. M., Brooks, T. F., Bahr, C. J., Spalt, T. B., Bartram, S. M., Culliton, W. G., and Becker, L. E., "Development of a Microphone Phased Array Capability for the Langley 14- by 22-Foot Subsonic Tunnel," AIAA 2014-2343, 20th AIAA/CEAS Aeroacoustics Conference, AIAA Aviation 2014, Atlanta, GA, June 2014.

${ }^{20}$ Horne, W. C. and Burnside, N. J., "AMELIA CESTOL Test: Acoustic Characteristics of Circulation Control Wing and Leading- and Trailing-Edge Slot Blowing," AIAA 2013-0978, 51st AIAA Aerospace Sciences Meeting, Dallas/Ft. Worth, TX, January 2013.

${ }^{21}$ Brooks, T. F. and Humphreys, W. M., "A deconvolution approach for the mapping of acoustic sources (DAMAS) determined from phased microphone arrays," Journal of Sound and Vibration, Vol. 294, 2006, pp. 856-879.
} 